\title{
CONVERGENT FINITE ELEMENT DISCRETIZATIONS \\ OF THE NONSTATIONARY INCOMPRESSIBLE MAGNETOHYDRODYNAMICS SYSTEM
}

\author{
AndReAs PROHL ${ }^{1}$
}

\begin{abstract}
The incompressible MHD equations couple Navier-Stokes equations with Maxwell's equations to describe the flow of a viscous, incompressible, and electrically conducting fluid in a Lipschitz domain $\Omega \subset \mathbb{R}^{3}$. We verify convergence of iterates of different coupling and decoupling fully discrete schemes towards weak solutions for vanishing discretization parameters. Optimal first order of convergence is shown in the presence of strong solutions for a splitting scheme which decouples the computation of velocity field, pressure, and magnetic fields at every iteration step.
\end{abstract}

Mathematics Subject Classification. 65N30.

Received March 10, 2008. Revised June 24, 2008.

Published online August 12, 2008.

\section{INTRODUCTION}

Let $\Omega \subset \mathbb{R}^{3}$ be a bounded polyhedral domain. The magneto-hydrodynamical behavior of liquid metals is described by the MHD equations

$$
\begin{array}{rlrl}
\mathbf{u}_{t}+(\mathbf{u} \cdot \nabla) \mathbf{u}-\frac{1}{\operatorname{Re}} \Delta \mathbf{u}+\nabla p+S \mathbf{b} \times \operatorname{curl} \mathbf{b} & =\mathbf{g} & & \text { in } \Omega_{T}:=(0, T) \times \Omega, \\
\operatorname{div} \mathbf{u}=0 \quad \operatorname{div} \mathbf{b}=0 & & \text { in } \Omega_{T}, \\
\mathbf{b}_{t}+\frac{1}{\operatorname{Re}_{m}} \operatorname{curl}(\operatorname{curl} \mathbf{b})-\operatorname{curl}(\mathbf{u} \times \mathbf{b}) & =\mathbf{0} & & \text { in } \Omega_{T},
\end{array}
$$

together with the following boundary and initial conditions,

$$
\begin{array}{llll}
\mathbf{u}=\mathbf{0}, & \mathbf{b} \cdot \mathbf{n}=0, & \operatorname{curl} \mathbf{b} \times \mathbf{n}=\mathbf{0} & \text { on } \partial \Omega_{T}:=(0, T) \times \partial \Omega, \\
& \mathbf{u}(0, \cdot)=\mathbf{u}_{0}, & \mathbf{b}(0, \cdot)=\mathbf{b}_{0} & \text { on } \Omega,
\end{array}
$$

where $\mathbf{n} \in \mathbb{R}^{3}$ denotes the outward normal unit vector on $\partial \Omega$. Three nondimensional numbers appear in (1.1)-(1.3), which are the Reynolds number Re $>0$, the magnetic Reynolds number $\operatorname{Re}_{m}>0$, and the coupling number $S=\frac{M^{2}}{\operatorname{ReRm}}>0$, where $M>0$ is the Hartman number. The system (1.1)-(1.3) couples the incompressible

\footnotetext{
Keywords and phrases. Magneto-hydrodynamics, discretization, FEM, fixed-point scheme, splitting-method.

1 Mathematisches Institut, Universität Tübingen, Auf der Morgenstelle 10, 72076 Tübingen, Germany.

prohl@na.uni-tuebingen.de
} 
Navier-Stokes equations with Maxwell's equations. By formally multiplying (1.1) with the velocity field $\mathbf{u}$, equation (1.2) with $S$ times the magnetic field $\mathbf{b}$, and integrating over $\Omega$, making the sum of both equations leads to the energy identity, where the energy ensembles kinetic and magnetic energy portions,

$$
\frac{1}{2} \frac{\mathrm{d}}{\mathrm{d} t} \int_{\Omega}\left[|\mathbf{u}|^{2}+S|\mathbf{b}|^{2}\right] \mathrm{d} \mathbf{x}+\int_{\Omega}\left[\frac{1}{\operatorname{Re}}|\nabla \mathbf{u}|^{2}+\frac{S}{\operatorname{Re}_{m}}|\operatorname{curl} \mathbf{b}|^{2}\right] \mathrm{d} \mathbf{x}=\int_{\Omega} \mathbf{g} \cdot \mathbf{u d} \mathbf{x} \quad \forall t>0 .
$$

A recent summary of known results for the MHD equations, including modeling, analysis, and numerics is [12]. Strong solutions to (1.1)-(1.3) are only known to exist for small times and sufficiently regular data, while weak solutions exist globally. This difference in notion of solution for (1.1)-(1.3) is reflected in constructing different numerical schemes. For stationary incompressible MHD equations, there are numerical analyses for schemes available, whose convergence is shown either in the context of existing weak or strong solutions; cf. e.g. [15,16,25], and [11].

The numerical analysis of the stationary incompressible MHD problem started with [15], where inf-sup stable mixed elements are used to discretize velocity fields and hydrostatic pressure, and $\mathbf{W}^{1,2}$-conforming elements for the magnetic field; as is shown in [15], Section 6, solutions of the nonlinear finite element scheme exist, and converge to weak solutions of the limiting problem, provided that $\Omega \subset \mathbb{R}^{3}$ is either a convex polyhedron or has a boundary which is $C^{1,1}$; see [15], Theorem 6.4. As is e.g. known from [9], see also [1,14], the magnetic field in non-convex polyhedra $\Omega$ may fail to be in $\mathbf{W}^{1,2}(\Omega)$, such that nodal finite element discretizations, albeit stable, may not converge to corresponding magnetization fields. This observation recently motivated the works [16,25], where a mixed formulation of the stationary incompressible MHD problem based on $\mathbf{H}$ (curl)conforming (edge) elements to approximate the magnetic field is proposed, and convergence to weak solutions of the limiting problem is shown for vanishing spatial discretization parameter [25], Corollary 4.1. A different strategy to achieve convergence in general polyhedral domains is realized in [16], where certain weight functions to account for possible singular solution components induced by reentrant vertices or edges complement a $\mathbf{W}^{1,2}(\Omega)$-conforming finite element discretization. For convex polyhedral domains, or domains with a boundary which is $C^{1,1}$, convergence of a stabilized finite element method based on continuous finite elements is shown in [11].

The goal of this work is to propose and analyze practical, fully discrete schemes which construct weak solutions of the evolutionary problem (1.1)-(1.5) for vanishing discretization parameters, as well as efficient space-time discretizations of (1.1)-(1.5) where iterates approximate regular strong solutions at optimal rates of convergence. Our focus is to compare the different requirements needed to theoretically validate convergence of the different schemes.

The remainder of this work is organized as follows. We introduce notation and recall necessary material in Section 2. A finite element based temporal discretization of (1.1)-(1.3) introduced in [2] is analyzed in Section 3, which inherits a discrete energy law to the fully discrete setting (Scheme A). The first main result is Theorem 3.1, which verifies that weak solutions of (1.1)-(1.3) may be obtained from corresponding iterates for vanishing discretization parameters. A disadvantage of Scheme A is the need to compute iterates for velocity and magnetic field in a coupled manner, which is cured in the following ways:

(i) A simple fixed point strategy is proposed to decouple the computation of iterates for velocity and magnetic field (Algorithm A): a restrictive mesh-size constraint enters as a sufficient constraint to validate convergence of the algebraic solver, and hence verify overall (subsequence) convergence towards weak solutions of (1.1)-(1.3); cf. Theorem 3.2.

(ii) Scheme B is a decoupled variant of Scheme A, where a discrete energy inequality holds only if $k=$ $\mathcal{O}\left(h^{3}\right)$ is valid. Overall (subsequence) convergence for vanishing discretization parameters towards weak solutions of (1.1)-(1.3) is then stated in Theorem 3.3.

These results hold under minimum assumptions with respect to given data, and proposed schemes construct weak solutions of (1.1)-(1.3) for vanishing discretization parameters in a fully practical manner. A different viewpoint is taken in Section 4, where optimal rates of convergence for iterates computed by the splitting Scheme C towards existing strong solutions of (1.1)-(1.3) are verified; $c f$. Theorem 4.1. This strategy allows for 
a fully decoupled computation of iterates of velocity, magnetic field, and pressure, and combines the decoupling strategy from Scheme B with the projection method of Chorin $[8,27]$. As is detailed in Section 4, the Scheme C is much easier to implement, and is based on stabilization strategies to approximate solenoidal velocity and magnetic fields. Conclusions are drawn in Section 5.

\section{PRELiminaries}

\subsection{Functional spaces and notion of weak solution for MHD equations}

Let $\Omega \subset \mathbb{R}^{3}$ be a bounded polyhedral domain. For simplicity, we assume that $\Omega$ is simply-connected, and that its boundary $\partial \Omega$ is connected. Let $\mathbf{H}(\operatorname{div} ; \Omega)$ ensemble vector fields $\boldsymbol{\xi} \in \mathbf{L}^{2}(\Omega)$, such that $\operatorname{div} \boldsymbol{\xi} \in L^{2}(\Omega)$; the space is endowed with the norm $\|\boldsymbol{\xi}\|_{\mathbf{H}(\text { div })}:=\left(\|\boldsymbol{\xi}\|_{\mathbf{L}^{2}}^{2}+\|\operatorname{div} \boldsymbol{\xi}\|_{L^{2}}^{2}\right)^{1 / 2}$. We introduce the following subspaces $\mathbf{H}_{0}(\operatorname{div} ; \Omega):=\{\boldsymbol{\xi} \in \mathbf{H}(\operatorname{div} ; \Omega): \boldsymbol{\xi} \cdot \mathbf{n}=0$ on $\partial \Omega\}$, and

$$
\begin{aligned}
\mathcal{J} & :=\left\{\boldsymbol{\xi} \in \mathbf{C}_{0}^{\infty}(\Omega): \operatorname{div} \boldsymbol{\xi}=0 \text { in } \Omega\right\} \\
\mathbf{H} & :=\left\{\boldsymbol{\xi} \in \mathbf{L}^{2}(\Omega): \operatorname{div} \boldsymbol{\xi}=0 \text { weakly in } \Omega, \text { and } \boldsymbol{\xi} \cdot \mathbf{n}=0\right\}, \\
\mathbf{J} & :=\left\{\boldsymbol{\xi} \in \mathbf{W}_{0}^{1,2}(\Omega): \operatorname{div} \boldsymbol{\xi}=0 \text { a.e. in } \Omega\right\} .
\end{aligned}
$$

By Helmholtz orthogonal decomposition, a vector field can be written as unique sum of its solenoidal and irrotational components, i.e., $\mathbf{L}^{2}(\Omega)=\mathbf{H} \oplus \nabla W^{1,2}(\Omega)$. Correspondingly, we introduce the space $\mathbf{H}(\operatorname{curl} ; \Omega)$, which is endowed with the norm $\|\boldsymbol{\psi}\|_{\mathbf{H}(\operatorname{curl})}:=\left(\|\boldsymbol{\psi}\|_{\mathbf{L}^{2}}^{2}+\|\operatorname{curl} \boldsymbol{\psi}\|_{\mathbf{L}^{2}}^{2}\right)^{1 / 2}$, and subspaces $\mathbf{H}_{0}(\operatorname{curl} ; \Omega):=\{\boldsymbol{\psi} \in$ $\mathbf{H}(\operatorname{curl} ; \Omega): \boldsymbol{\psi} \times \mathbf{n}=0$ on $\partial \Omega\}$, as well as

$$
\begin{aligned}
\mathcal{W} & :=\left\{\boldsymbol{\psi} \in \mathbf{C}^{\infty}(\bar{\Omega}): \operatorname{div} \boldsymbol{\psi}=0 \text { in } \Omega, \boldsymbol{\psi} \cdot \mathbf{n}=0 \text { on } \partial \Omega\right\} \\
\mathcal{H} & :=\mathbf{H}(\operatorname{curl} ; \Omega) \cap \mathbf{H}_{0}(\operatorname{div} ; \Omega) \\
\mathbf{X} & :=\{\boldsymbol{\psi} \in \mathcal{H}: \operatorname{div} \boldsymbol{\psi}=0 \text { a.e. in } \Omega\} .
\end{aligned}
$$

The space $\mathcal{D}(\bar{\Omega})$ of restrictions to $\bar{\Omega}$ of smooth functions $\mathcal{D}\left(\mathbb{R}^{3}\right)$ is dense, both in $\mathbf{H}(\operatorname{div} ; \Omega)$ and $\mathbf{H}(\operatorname{curl} ; \Omega)$; cf. [1], Proposition 2.3. For all $\boldsymbol{\psi} \in \mathbf{X}$, we have Poincare's inequality $\|\boldsymbol{\psi}\|_{\mathbf{L}^{2}} \leq C \|$ curl $\boldsymbol{\psi} \|_{\mathbf{L}^{2}}$ for all $\boldsymbol{\psi} \in \mathbf{X}$, which implies that $\|\cdot\|_{\mathbf{H}(\text { curl) }}$ and $\|\operatorname{curl}(\cdot)\|_{\mathbf{L}^{2}}$ are equivalent norms on $\mathbf{X}$. The space $\mathbf{H}_{0}(\operatorname{curl} ; \Omega) \cap \mathbf{H}_{0}(\operatorname{div} ; \Omega)$ coincides with $\mathbf{W}_{0}^{1,2}(\Omega)$; in contrast, explicit counterexamples illustrate that $\mathbf{H}_{0}(\operatorname{curl} ; \Omega)$ and $\mathbf{H}_{0}(\operatorname{div} ; \Omega)$ are continuously embedded into $\mathbf{W}^{1,2}(\Omega)$ only if $\Omega \subset \mathbb{R}^{3}$ is either a convex polyhedron or has a boundary which is $C^{1,1} ; c f$. [14], Section I.3.5, or [1], Section 2.3. The embeddings of $\mathbf{H}(\operatorname{curl} ; \Omega)$ and $\mathbf{H}(\operatorname{div} ; \Omega)$ into $\mathbf{L}^{2}(\Omega)$ are not compact; however, Weber verified compactness of $\mathbf{H}_{0}(\operatorname{curl} ; \Omega)$ and $\mathbf{H}_{0}(\operatorname{div} ; \Omega)$ into $\mathbf{L}^{2}(\Omega)$ for general Lipschitz polyhedral domains; the following compactness results may be found in [1], Proposition 3.7, and [25], Proposition 2.3.

Lemma 2.1. Let $\Omega \subset \mathbb{R}^{3}$ be a Lipschitz polyhedron.

(i) There exists an exponent $s \equiv s(\Omega)>\frac{1}{2}$, such that $\mathcal{H} \hookrightarrow \mathbf{W}^{s, 2}(\Omega)$ is continuous.

(ii) There exists a parameter $\delta_{1} \equiv \delta_{1}(\Omega)>0$ such that the embedding $\mathcal{H} \hookrightarrow \mathbf{L}^{3+\delta_{1}}(\Omega)$ is compact.

We need the Aubin-Lions' compactness result for Bochner spaces, $c f$. [12], Lemma 2.8.

Lemma 2.2. Let $B$ be a Banach space, and $B_{0}$ and $B_{1}$ be two reflexive Banach spaces. Assume $B_{0} \Subset B \subset B_{1}$. Fix $T<\infty$, and $1<p_{0}, p_{1}<\infty$. Then

$$
\left\{v \in L^{p_{0}}\left(0, T ; B_{0}\right): \partial_{t} v \in L^{p_{1}}\left(0, T ; B_{1}\right)\right\} \Subset L^{p_{0}}(0, T ; B) .
$$




\subsection{Weak solutions for the MHD equations}

We introduce the notion of weak solutions to (1.1)-(1.3) on bounded polyhedral domains $\Omega \subset \mathbb{R}^{3}$.

Definition 2.1. Let $\mathbf{g} \in L^{2}\left(0, T ; \mathbf{J}^{\prime}\right)$, and $\mathbf{u}_{0}, \mathbf{b}_{0} \in \mathbf{H}$. The pair $(\mathbf{u}, \mathbf{b})$ is said to be a weak solution of (1.1)-(1.5), if

(i) $\mathbf{u} \in L^{\infty}(0, T ; \mathbf{H}) \cap L^{2}(0, T ; \mathbf{J})$, and $\mathbf{b} \in L^{\infty}(0, T ; \mathbf{H}) \cap L^{2}(0, T ; \mathbf{X})$;

(ii) $(\mathbf{u}, \mathbf{b})$ is weakly continuous for $t>0$, with values in $\mathbf{H}$;

(iii) for every $(\boldsymbol{\xi}, \boldsymbol{\psi}) \in C_{0}^{\infty}([0, T) ; \mathcal{J}) \times C_{0}^{\infty}([0, T) ; \boldsymbol{W})$, there hold

$$
\begin{aligned}
& \int_{0}^{T}\left[-\left(\mathbf{u}, \boldsymbol{\xi}_{t}\right)+\frac{1}{\operatorname{Re}}(\nabla \mathbf{u}, \nabla \boldsymbol{\xi})-((\mathbf{u} \cdot \nabla) \boldsymbol{\xi}, \mathbf{u})+S(\mathbf{b} \times \operatorname{curl} \mathbf{b}, \boldsymbol{\xi})\right] \mathrm{d} t=\left(\mathbf{u}_{0}, \boldsymbol{\xi}\right)+\int_{0}^{T}(\mathbf{g}, \boldsymbol{\xi}) \mathrm{d} t \\
& \int_{0}^{T}\left[-\left(\mathbf{b}, \boldsymbol{\psi}_{t}\right)+\frac{1}{\operatorname{Re}_{m}}(\operatorname{curl} \mathbf{b}, \operatorname{curl} \boldsymbol{\psi})-(\mathbf{u} \times \mathbf{b}, \operatorname{curl} \boldsymbol{\psi})\right] \mathrm{d} t=\left(\mathbf{b}_{0}, \boldsymbol{\psi}\right) ;
\end{aligned}
$$

(iv) for almost every $t \in[0, T]$,

$$
\begin{aligned}
\frac{1}{2}\left[\|\mathbf{u}(t, \cdot)\|_{\mathbf{L}^{2}}^{2}+S\|\mathbf{b}(t, \cdot)\|_{\mathbf{L}^{2}}^{2}\right]+\int_{0}^{t}\left[\frac{1}{\operatorname{Re}}\|\nabla \mathbf{u}\|_{\mathbf{L}^{2}}^{2}+\frac{S}{\operatorname{Re}_{m}}\|\operatorname{curl} \mathbf{b}\|_{\mathbf{L}^{2}}^{2}\right] \mathrm{d} s \leq & \leq \\
& \frac{1}{2}\left[\left\|\mathbf{u}_{0}\right\|_{\mathbf{L}^{2}}^{2}+S\left\|\mathbf{b}_{0}\right\|_{\mathbf{L}^{2}}^{2}\right]+\int_{\Omega_{t}} \mathbf{g} \cdot \mathbf{u} \mathrm{d} \mathbf{x} \mathrm{d} s .
\end{aligned}
$$

In [12], Chapter 2, weak solutions for $\Omega \subset \mathbb{R}^{3}$ a convex polyhedron, or a domain with $C^{1,1}$-boundary are constructed by a general Galerkin method which uses eigenvalues of the Stokes operator $\mathcal{A}_{1}: \mathbf{J} \rightarrow \mathbf{J}^{\prime}$, and the magnetostatic operator $\mathcal{A}_{2}: \mathbf{X} \rightarrow \mathbf{X}^{\prime}$. Here, $\mathcal{A}_{1} \mathbf{u}=\mathbf{f} \in \mathbf{J}^{\prime}$ if

$$
(\nabla \mathbf{u}, \nabla \boldsymbol{\xi})-(p, \operatorname{div} \boldsymbol{\xi})=(\mathbf{f}, \boldsymbol{\xi}) \quad \forall \boldsymbol{\xi} \in \mathbf{W}_{0}^{1,2}(\Omega)
$$

and $\mathcal{A}_{2} \mathbf{b}=\mathbf{g} \in \mathbf{X}^{\prime}$ if

$$
(\operatorname{curl} \mathbf{b}, \operatorname{curl} \boldsymbol{\psi})=(\mathrm{g}, \boldsymbol{\psi}) \quad \forall \boldsymbol{\psi} \in \mathbf{X} \text {. }
$$

In this paper, weak solutions for general bounded polyhedral domains $\Omega \subset \mathbb{R}^{3}$ are obtained by a practicable discretization of (1.1)-(1.5) which uses finite elements.

\subsection{Strong solutions to MHD equations}

Strong solutions for (1.1)-(1.5) are weak solutions of the MHD equations for $\mathbf{g} \in L^{2}(0, T ; \mathbf{H})$, and $\left(\mathbf{u}_{0}, \mathbf{b}_{0}\right) \in$ $\mathbf{J} \times \mathbf{X}$ such that

$$
\mathbf{u} \in L^{\infty}(0, T ; \mathbf{J}) \cap L^{2}\left(0, T ; \mathbf{J} \cap \mathbf{W}^{2,2}(\Omega)\right), \quad \mathbf{b} \in L^{\infty}(0, T ; \mathbf{X}) \cap L^{2}\left(0, T ; \mathbf{X} \cap \mathbf{W}^{2,2}(\Omega)\right)
$$

As an immediate consequence, we obtain bounds for distributional time-derivatives of solutions of (1.1)-(1.5), and hence may validate $(\mathbf{u}, \mathbf{b}) \in C([0, T] ; \mathbf{J}) \times C([0, T] ; \mathbf{X})$. Local, unique strong solutions have been constructed in [26], Theorem 3.2 for bounded domains $\Omega \subset \mathbb{R}^{3}$ with regular boundary $\partial \Omega$; necessary tools are regularity properties of solutions $(\mathbf{u}, \mathbf{b}) \in \mathbf{J} \times \mathbf{X}$ of $(2.1)$ and $(2.2)$, for $(\mathbf{f}, \mathbf{g}) \in\left[\mathbf{L}^{2}(\Omega)\right]^{2}$,

$$
(\mathbf{u}, p) \in \mathbf{W}^{2,2}(\Omega) \times\left[W^{1,2}(\Omega) \cap L_{0}^{2}(\Omega)\right], \quad \text { where } \quad\|\mathbf{u}\|_{\mathbf{W}^{2,2}}+\|p\|_{L_{0}^{2}} \leq C\|\mathbf{f}\|_{\mathbf{L}^{2}}
$$

by Cattabriga [6] (see also [14], Thm. 5.4 and Rem. 5.6) for some finite, positive $C \equiv C(\Omega)$, and likewise

$$
\mathbf{b} \in \mathbf{W}^{2,2}(\Omega), \quad \text { where } \quad\|\mathbf{b}\|_{\mathbf{W}^{2,2}} \leq C\|\mathbf{g}\|_{\mathbf{L}^{2}}
$$


by Georgescu [10], Theorem 3.2.2 (see also [12], Thm. 2.24]). By using $\operatorname{curl} \operatorname{curl} \varphi=\operatorname{grad} \operatorname{div} \varphi-\Delta \varphi$ for all $\varphi \in \mathbf{W}^{1,2}(\Omega)$ we have that a solution $\mathbf{b} \in \mathbf{W}^{1,2}(\Omega)$ of (2.2) satisfies in distributional sense

$$
-\Delta \mathbf{b}=\mathbf{g} \quad \text { in } \Omega, \quad \mathbf{b} \cdot \mathbf{n}=0 \quad \text { and } \quad \operatorname{curl} \mathbf{b} \times \mathbf{n}=\mathbf{0} \quad \text { on } \partial \Omega .
$$

\subsection{Finite element spaces}

Let $\mathcal{T}_{h}$ be a quasi-uniform triangulation of the polygonal resp. polyhedral domain $\Omega \subset \mathbb{R}^{3}$ into tetrahedra of maximal diameter $h>0$, i.e., $\bar{\Omega}=\bigcup_{K \in \mathcal{T}_{h}} \bar{K}$. Let $V_{h}:=\left\{\xi \in C_{0}(\bar{\Omega}): \xi \in P_{i}(K) \forall K \in \mathcal{T}_{h}\right\}$, where $P_{i}$ ensembles polynomials of degree less or equal $i \geq 1$. We recall the inverse inequality [5], Lemma 4.5.3, which holds for $\xi \in V_{h}$,

$$
\|\xi\|_{L^{\ell, q_{1}}} \leq C h^{m-\ell+n \min \left\{\frac{1}{q_{1}}-\frac{1}{q_{2}}, 0\right\}}\|\xi\|_{W^{m, q_{2}}} \quad \forall 1 \leq q_{1}, q_{2} \leq \infty, \quad 0 \leq m \leq \ell .
$$

Let $\mathbf{V}_{h}:=\left[V_{h}\right]^{3}$. We define $\mathbf{L}^{2}(\Omega)$-, resp. $\mathbf{W}_{0}^{1,2}(\Omega)$-orthonormal projections $\mathbf{P}_{h}^{0}$ resp. $\mathbf{P}_{h}^{1}$ to $\mathbf{V}_{h}$,

$$
\left(\mathbf{z}-\mathbf{P}_{h}^{0} \mathbf{z}, \boldsymbol{\xi}\right)=0 \quad \forall \boldsymbol{\xi} \in \mathbf{V}_{h}, \quad \text { resp. } \quad\left(\nabla\left[\mathbf{z}-\mathbf{P}_{h}^{1} \mathbf{z}\right], \nabla \boldsymbol{\xi}\right)=0 \quad \forall \boldsymbol{\xi} \in \mathbf{V}_{h} .
$$

Let $\mathcal{E}_{h}=\left\{\mathbf{x}_{\ell}\right\}_{\ell \in L}$ denote the set of all nodes of $\mathcal{T}_{h}$. We define the nodal interpolation operator $\mathcal{I}_{\mathbf{V}_{h}}$ : $C\left(\bar{\Omega}, \mathbb{R}^{3}\right) \rightarrow \mathbf{V}_{h}$, such that $\mathcal{I}_{\mathbf{V}_{h}} \xi:=\sum_{\mathbf{z} \in \mathcal{E}_{h}} \xi(\mathbf{z}) \varphi_{\mathbf{z}}$, where $\left\{\varphi_{\mathbf{z}}: \mathbf{z} \in \mathcal{E}_{h}\right\}$ denotes the nodal basis of $V_{h}$.

For $j \geq 0$, consider $L_{h}:=\left\{\Pi \in L_{0}^{2}(\Omega): \Pi \in P_{j}(K) \forall K \in \mathcal{T}_{h}\right\}$. The spaces $\left(\mathbf{V}_{h}, L_{h}\right)$ are chosen such that the discrete LBB-condition holds, i.e., $\mathbf{V}_{h} \subset \mathbf{W}_{0}^{1,2}\left(\Omega, \mathbb{R}^{3}\right)$ and $L_{h} \subset L_{0}^{2}(\Omega)$ satisfy the discrete inf-sup condition

$$
\sup _{\boldsymbol{\xi} \in \mathbf{V}_{h}} \frac{(\operatorname{div} \boldsymbol{\xi}, \Pi)}{\|\nabla \boldsymbol{\xi}\|_{\mathbf{L}^{2}}} \geq C\|\Pi\|_{L^{2}} \quad \forall \Pi \in L_{h}
$$

Let

$$
\mathbf{J}_{h}:=\left\{\boldsymbol{\xi} \in \mathbf{V}_{h}:(\operatorname{div} \boldsymbol{\xi}, \Pi)=0 \text { for all } \Pi \in L_{h}\right\} \not \subset \mathbf{J}
$$

be the space of discretely solenoidal functions. Let $\mathbf{u} \in \mathbf{J}$ be a solution of $\mathcal{A}_{1} \mathbf{u}=\mathbf{f}$ in $\Omega \subset \mathbb{R}^{3}$. The solution $(\mathbf{U}, P) \in \mathbf{J}_{h} \times L_{h}$ of the discretized incompressible Stokes problem

$$
(\nabla \mathbf{U}, \nabla \boldsymbol{\xi})-(P, \operatorname{div} \boldsymbol{\xi})=(\mathbf{f}, \boldsymbol{\xi}) \quad \forall \boldsymbol{\xi} \in \mathbf{V}_{h}
$$

satisfies

We define $\mathbf{L}^{2}$ - resp. $\mathbf{W}_{0}^{1,2}$-orthogonal projections to $\mathbf{J}_{h}$ via

$$
\|\mathbf{u}-\mathbf{U}\|_{\mathbf{L}^{2}}+h\|\mathbf{u}-\mathbf{U}\|_{\mathbf{W}^{1,2}} \leq C h^{2}\|\mathbf{u}\|_{\mathbf{W}^{2,2} .}
$$

$$
\left(\mathbf{z}-\mathbf{Q}_{h}^{0} \mathbf{z}, \boldsymbol{\xi}\right)=0 \quad \forall \boldsymbol{\xi} \in \mathbf{J}_{h} \quad \text { resp. } \quad\left(\nabla\left[\mathbf{z}-\mathbf{Q}_{h}^{1} \mathbf{z}\right], \nabla \boldsymbol{\xi}\right)=0 \quad \forall \boldsymbol{\xi} \in \mathbf{J}_{h} .
$$

The following estimates follow from the above error estimates (see also [17]) $(i=0,1)$,

$$
\begin{aligned}
\left\|\mathbf{z}-\mathbf{Q}_{h}^{i} \mathbf{z}\right\|_{\mathbf{L}^{2}}+h\left\|\nabla\left[\mathbf{z}-\mathbf{Q}_{h}^{i} \mathbf{z}\right]\right\|_{\mathbf{L}^{2}} & \leq C h^{2}\|\mathbf{z}\|_{\mathbf{W}^{2,2}} \quad \forall \mathbf{z} \in \mathbf{J} \cap \mathbf{W}^{2,2}(\Omega), \\
\left\|\mathbf{z}-\mathbf{Q}_{h}^{i} \mathbf{z}\right\|_{\mathbf{L}^{2}} & \leq C h\|\mathbf{z}\|_{\mathbf{W}^{1,2}} \quad \forall \mathbf{z} \in \mathbf{J} \cap \mathbf{W}^{1,2}(\Omega) .
\end{aligned}
$$

The following stability bound holds for all $2 \leq r \leq \infty$, see [13],

$$
\left\|\nabla \mathbf{Q}_{h}^{1} \mathbf{z}\right\|_{\mathbf{L}^{r}} \leq C\|\nabla \mathbf{z}\|_{\mathbf{L}^{r}} \quad \forall \mathbf{z} \in \mathcal{J} \cap \mathbf{W}_{0}^{1, r}(\Omega) .
$$

To approximate solenoidal solutions of Maxwell's equation by nodal elements often introduces nonphysical artifacts [4,9]; cf. also [18], Section 4.5. This does not occur for edge elements, which only enforce continuity of 
tangential field components across inter-element boundaries. In below, we use Nédélec's first family of spaces (cf. [21]) for the unknown $\mathbf{B}: \Omega_{T} \rightarrow \mathbb{R}^{3}$. For every $K \in \mathcal{T}_{h}$ and $k \geq 1$, let $\mathcal{N}_{k}(K)=\left[\mathbf{P}_{k-1}(K)\right]^{3} \oplus \mathbf{D}_{k}(K)$, where $\mathbf{D}_{k}$ is given by the homogeneous polynomials $\mathbf{p}$ of degree $k$ that satisfy $\mathbf{p}(\mathbf{x}) \cdot \mathbf{x}=0$ on $K \in \mathcal{T}_{h}$. Consider then

$$
\begin{aligned}
\mathbf{C}_{h} & =\left\{\boldsymbol{\psi} \in \mathbf{H}(\operatorname{curl}): \boldsymbol{\psi} \in \mathcal{N}_{k}(K) \quad \forall K \in \mathcal{T}_{h}\right\} \\
S_{h} & =\left\{R \in W^{1,2}(\Omega) \cap L_{0}^{2}(\Omega): R \in \mathrm{P}_{k}(K) \quad \forall K \in \mathcal{T}_{h}\right\}
\end{aligned}
$$

where $\mathbf{C}_{h} \subset \mathbf{H}(\operatorname{curl} ; \Omega)$, and $S_{h} \subset W^{1,2}(\Omega) \cap L_{0}^{2}(\Omega)$. A well-known example is $k=1$, where $\mathbf{C}_{h}=\{\boldsymbol{\psi} \in \mathbf{H}($ curl $)$ : $\psi=\mathbf{a}_{K}+\mathbf{b}_{K} \times \mathbf{x}$ on $\left.K \forall K \in \mathcal{T}_{h}\right\}$. The spaces $\left(\mathbf{C}_{h}, S_{h}\right)$ satisfy the following discrete inf-sup condition,

$$
\sup _{\boldsymbol{\psi}_{h} \in \mathbf{C}_{h}} \frac{(\nabla R, \boldsymbol{\psi})}{\|\boldsymbol{\psi}\|_{\mathbf{H}(\mathrm{curl})}} \geq C\|\nabla R\|_{L^{2}} \quad \forall R \in S_{h} .
$$

We may define the interpolation $\boldsymbol{\mathcal { R }}_{h}: \mathbf{H}(\operatorname{curl} ; \Omega) \rightarrow \mathbf{C}_{h}$, which satisfies for all $\mathbf{z} \in\left\{\overline{\mathbf{z}} \in \mathbf{W}^{1,2}: \operatorname{curl} \overline{\mathbf{z}} \in \mathbf{W}^{1,2}\right\}$ that ([21], Thm. 5.41)

$$
\left\|\mathbf{z}-\boldsymbol{\mathcal { R }}_{h} \mathbf{z}\right\|_{\mathbf{L}^{2}}+\left\|\operatorname{curl}\left[\mathbf{z}-\mathcal{R}_{h} \mathbf{z}\right]\right\|_{\mathbf{L}^{2}} \leq C h\left(\|\mathbf{z}\|_{\mathbf{W}^{1,2}}+\|\operatorname{curl} \mathbf{z}\|_{\mathbf{W}^{1,2}}\right) .
$$

We introduce the discretely solenoidal function space

$$
\mathbf{X}_{h}:=\left\{\boldsymbol{\psi} \in \mathbf{C}_{h}:(\boldsymbol{\psi}, \nabla R)=0 \text { for all } R \in S_{h}\right\} \not \subset \mathbf{X} .
$$

We have the $L^{2}(\Omega)$-orthogonal discrete Helmholtz decomposition $\mathbf{C}_{h}=\mathbf{X}_{h} \oplus \nabla S_{h}$, as well as the discrete Poincare-Friedrichs inequality $\|\boldsymbol{\psi}\|_{L^{2}} \leq C\|\operatorname{curl} \psi\|_{L^{2}}$, for all $\boldsymbol{\psi} \in \mathbf{X}_{h} ; c f$. [21], Theorem 4.7. We introduce the $\mathbf{L}^{2}(\Omega)$ - resp. $\mathbf{H}(\operatorname{curl} ; \Omega)$-orthogonal projections to $\mathbf{X}_{h}$ via

$$
\left(\mathbf{z}-\mathbf{R}_{h}^{0} \mathbf{z}, \boldsymbol{\psi}\right)=0 \quad \forall \boldsymbol{\psi} \in \mathbf{X}_{h} \quad \text { resp. } \quad\left(\operatorname{curl}\left[\mathbf{z}-\mathbf{R}_{h}^{1} \mathbf{z}\right], \operatorname{curl} \boldsymbol{\psi}\right)=0 \quad \forall \boldsymbol{\psi} \in \mathbf{X}_{h} .
$$

The following estimates may be found in [7], Theorem 3.5, or [28], Lemma 3.4, and [18], Theorem 4.8,

$$
\begin{aligned}
& \left\|\mathbf{z}-\mathbf{R}_{h}^{i} \mathbf{z}\right\|_{\mathbf{L}^{2}}+\left\|\operatorname{curl}\left[\mathbf{z}-\mathbf{R}_{h}^{1} \mathbf{z}\right]\right\|_{\mathbf{L}^{2}} \leq C h\left(\|\mathbf{z}\|_{\mathbf{W}^{1,2}}+\|\operatorname{curl} \mathbf{z}\|_{\mathbf{W}^{1,2}}\right) \quad \forall \mathbf{z} \in \mathbf{X} \cap \mathbf{W}^{2,2}(\Omega), \\
& \left\|\mathbf{z}-\mathbf{R}_{h}^{i} \mathbf{z}\right\|_{\mathbf{L}^{2}} \leq C h^{\sigma}\|\operatorname{curl} \mathbf{z}\|_{\mathbf{L}^{2}} \quad \forall \mathbf{z} \in \mathbf{X}, \quad \text { for some } \sigma=\sigma(\Omega)>0 .
\end{aligned}
$$

Another link between the spaces $\mathbf{X}_{h}$ and $\mathbf{X}$ is accomplished by the Hodge mapping $\mathbf{H}: \mathbf{H}($ curl; $\Omega) \rightarrow \mathbf{X}$, such that

$$
\operatorname{curl} \mathbf{H}(\psi)=\operatorname{curl} \psi \quad \forall \boldsymbol{\psi} \in \mathbf{H}(\operatorname{curl} ; \Omega) .
$$

The following approximation property is shown in [18], Lemma 4.5,

$$
\|\mathbf{z}-\mathbf{H}(\mathbf{z})\|_{\mathbf{L}^{2}} \leq C h^{\frac{1}{2}+\sigma}\|\operatorname{curl} \mathbf{z}\|_{\mathbf{L}^{2}} \quad \forall \mathbf{z} \in \mathbf{X}_{h}, \quad \text { for some } \sigma=\sigma(\Omega)>0 .
$$

Lowest-order edge elements are divergence free inside each element. However, a nonzero divergence in distributional sense arises from discontinuities of the normal component at inter-element boundaries. Since $\mathbf{X}_{h} \not \subset \mathbf{X}$, the compactness of bounded sequences $\left\{\boldsymbol{\psi}_{h}\right\}_{h} \subset \mathbf{X}_{h} \subset \mathbf{H}(\operatorname{curl} ; \Omega)$ is not clear. The following (discrete) compactness property for discretely divergence-free vector fields generalizes the result by Kikuchi in [20]; cf. [18], Theorem 4.9.

Lemma 2.3. Let $\left\{\boldsymbol{\psi}_{h}\right\} \subset \mathbf{X}_{h}$ be a sequence of fields, which is uniformly bounded in $\mathbf{H}(\mathrm{curl} ; \Omega)$. Then there exists a subsequence $\left\{\boldsymbol{\psi}_{h^{\prime}}\right\}_{h^{\prime}}$ converging weakly in $\mathbf{H}(\operatorname{curl} ; \Omega)$, and strongly in $\mathbf{L}^{2}(\Omega)$ to a solenoidal function $\psi \in \mathbf{X}$. 


\subsection{Time discretization}

Given a time-step size $k>0$, and a sequence $\left\{\varphi^{j}\right\}$ in some Banach space $X$, we set $d_{t} \varphi^{j}:=k^{-1}\left\{\varphi^{j}-\varphi^{j-1}\right\}$ for $j \geq 1$. Note that $\left(d_{t} \varphi^{j}, \varphi^{j}\right)=\frac{1}{2} d_{t}\left\|\varphi^{j}\right\|^{2}+\frac{k}{2}\left\|d_{t} \varphi^{j}\right\|^{2}$, if $X$ is a Hilbert space. Piecewise constant interpolations of $\left\{\varphi^{j}\right\}$ are defined for $t \in\left[t_{j}, t_{j+1}\right)$, and $0 \leq j \leq J-1$ by

$$
\varphi^{+}(t):=\varphi^{j+1}, \quad \varphi^{-}(t):=\varphi^{j}
$$

and a piecewise affine interpolation on $\left[t_{j}, t_{j+1}\right)$ is defined by

$$
\varphi(t):=\frac{t-t_{j}}{k} \varphi^{j+1}+\frac{t_{j+1}-t}{k} \varphi^{j} .
$$

There holds $\left\|\varphi^{+}-\varphi\right\|_{X}+\left\|\varphi^{-}-\varphi\right\|_{X} \leq 2 k\left\|d_{t} \varphi\right\|_{X}$.

Consider the Navier-Stokes equations for an incompressible, viscous Newtonian fluid, by formally putting $S=0$ in (1.1). Projection methods are efficient time-discretization strategies [8,22,24,27], where iterates for velocity field and pressure are independently computed at every time-step. The following projection scheme by Chorin is well-known to compute iterates $\left(\mathbf{u}^{n}, p^{n}\right) \in \mathbf{H} \times L_{0}^{2}(\Omega)$ from a given $\mathbf{u}^{n-1} \in \mathbf{H}$, for every $n \geq 1[8,27]$ :

1. Let $n \geq 1$. Compute $\tilde{\mathbf{u}}^{n} \in \mathbf{W}_{0}^{1,2}(\Omega)$ from

$$
\frac{1}{k}\left(\tilde{\mathbf{u}}^{n}-\mathbf{u}^{n-1}\right)-\frac{1}{\operatorname{Re}} \Delta \tilde{\mathbf{u}}^{n}+\left[\mathbf{u}^{n-1} \cdot \nabla\right] \tilde{\mathbf{u}}^{n}=\mathbf{g}^{n} \quad \text { in } \Omega
$$

2. Compute $\left(\mathbf{u}^{n}, p^{n}\right) \in \mathbf{H} \times L_{0}^{2}(\Omega)$ from

$$
\frac{1}{k}\left(\mathbf{u}^{n}-\tilde{\mathbf{u}}^{n}\right)+\nabla p^{n}=\mathbf{0} \quad \text { in } \Omega .
$$

The latter step can be reformulated as a problem for the pressure function only,

$$
-\Delta p^{n}=-\frac{1}{k} \operatorname{div} \tilde{\mathbf{u}}^{n} \quad \text { in } \Omega, \quad \partial_{\mathbf{n}} p=0 \quad \text { on } \partial \Omega
$$

Hence, each step consists of a decoupled computation of $\tilde{\mathbf{u}}^{n} \in \mathbf{W}_{0}^{1,2}(\Omega), p^{n} \in W^{1,2}(\Omega) \cap L_{0}^{2}(\Omega)$, and $\mathbf{u}^{n} \in \mathbf{H}$ comes from a simple algebraic update.

Inherent error effects due to temporal discretization, and splitting resp. decoupling, which goes along with unphysical boundary conditions for pressure iterates may be understood by the following reformulation,

$$
\begin{aligned}
d_{t} \tilde{\mathbf{u}}^{n}-\frac{1}{\operatorname{Re}} \Delta \tilde{\mathbf{u}}^{n}+\left[\mathbf{P}_{\mathbf{H}} \tilde{\mathbf{u}}^{n-1} \cdot \nabla\right] \tilde{\mathbf{u}}^{n}+\nabla p^{n-1} & =\mathbf{g}^{n}, \\
\operatorname{div} \tilde{\mathbf{u}}^{n}-k \Delta p^{n} & =0, \quad \text { and } \quad \partial_{\mathbf{n}} p^{n}=0 \quad \text { on } \partial \Omega .
\end{aligned}
$$

It is due to the decoupling character of the projection method that no discrete energy law is available. However, for strong solutions

$$
(\mathbf{u}, p) \in L^{\infty}\left(0, T ; \mathbf{J} \cap \mathbf{W}^{2,2}(\Omega)\right) \times L^{\infty}\left(0, T ; W^{1,2}(\Omega) \cap L_{0}^{2}(\Omega)\right)
$$

which exist at least locally in time on regular domains $\Omega \subset \mathbb{R}^{3}$ for $\mathbf{u}_{0} \in \mathbf{J} \cap \mathbf{W}^{2,2}(\Omega)$ (cf. Sect. 2.3), the following result is shown in [22], Chapter 6. We need two assumptions (A1), (A2) with respect to regularity of data, which are detailed in Section 4.

Lemma 2.4. Let $\Omega \subset \mathbb{R}^{3}$, and suppose (A1), (A2). Let $\left\{\left(\tilde{\mathbf{u}}^{n}, p^{n}\right)\right\}_{n=1}^{N} \in \mathbf{W}_{0}^{1,2}(\Omega) \times\left[W^{1,2}(\Omega) \cap L_{0}^{2}(\Omega)\right]$ be solutions of $(2.15)-(2.16)$, and

$$
\left\|\mathbf{u}^{0}-\mathbf{u}_{0}\right\|_{\mathbf{L}^{2}}+\sqrt{k}\left\|\mathbf{u}^{0}-\mathbf{u}_{0}\right\|_{\mathbf{W}^{1,2}} \leq C k .
$$


For sufficiently small time-steps $k \leq k_{0}\left(\Omega_{T}, \mathrm{Re}\right)$, there exists a constant $C \equiv C\left(\Omega_{T}, \mathrm{Re}\right)>0$ such that $\left(\tau^{n}:=\right.$ $\left.\min \left\{1, t_{n}\right\}\right)$

$$
\begin{aligned}
\frac{1}{2} \max _{1 \leq n \leq N}\left[\left\|\mathbf{u}\left(t_{n}, \cdot\right)-\tilde{\mathbf{u}}^{n}\right\|_{\mathbf{L}^{2}}^{2}+\left(\tau^{n} \| p\left(t_{n}, \cdot\right)\right.\right. & \left.\left.-p^{n} \|_{W^{-1,2}}\right)^{2}+\left(\sqrt{\tau^{n} k}\left\|p\left(t_{n}, \cdot\right)-p^{n}\right\|_{L_{0}^{2}}\right)^{2}\right] \\
& +\frac{k^{2}}{2} \sum_{n=1}^{N}\left\|d_{t}\left[\mathbf{u}\left(t_{n}, \cdot\right)-\tilde{\mathbf{u}}^{n}\right]\right\|_{\mathbf{L}^{2}}^{2}+\frac{k}{\operatorname{Re}} \sum_{n=1}^{N}\left\|\nabla\left[\mathbf{u}\left(t_{n}, \cdot\right)-\tilde{\mathbf{u}}^{n}\right]\right\|_{\mathbf{L}^{2}}^{2} \leq C k^{2} .
\end{aligned}
$$

Moreover, the following uniform a-priori bounds are valid,

$$
\max \left[\left\|d_{t} \tilde{\mathbf{u}}^{n}\right\|_{\mathbf{L}^{2}}+\left\|\tilde{\mathbf{u}}^{n}\right\|_{\mathbf{W}^{2,2}}+\left\|p^{n}\right\|_{W^{1,2} \cap L_{0}^{2}}\right]+\left(k \sum_{n=1}^{N}\left[\left\|d_{t} \tilde{\mathbf{u}}^{n}\right\|_{\mathbf{W}^{1,2}}^{2}+\left\|d_{t} p^{n}\right\|_{L_{0}^{2}}^{2}\right]\right)^{1 / 2} \leq C .
$$

Here, $W^{-1,2}(\Omega)=\left[W_{0}^{1,2}(\Omega)\right]^{\prime}$, and suboptimal error estimates for the pressure in $L^{2}(\Omega)$ vs. optimal ones in $W^{-1,2}(\Omega)$ reflect arising boundary layers, which are due to the unphysical boundary data prescribed for the pressure in $(2.18)_{2}$. We refer to [24] for a sharp local analysis of errors for the pressure in the context of strong solutions.

\section{Reliable Discretizations With DiscRete EnERgy PRINCIPle}

We use a discrete Lagrange multiplier $R^{n} \subset S_{h}$ to enforce $\mathbf{B}^{n}: \Omega \rightarrow \mathbb{R}^{3}$ to be discretely solenoidal. Let $k>0$ be the equidistant time-discretization parameter, and $\mathcal{T}_{h}$ be a quasi-uniform triangulation of the bounded domain $\Omega \subset \mathbb{R}^{3}$. We set $\left(\mathbf{U}^{0}, \mathbf{B}^{0}\right)=\left(\mathbf{Q}_{h}^{0} \mathbf{u}_{0}, \mathbf{Q}_{h}^{0} \mathbf{b}^{0}\right)$.

Scheme A. Let $n \geq 1$. Find $\left(\mathbf{U}^{n}, P^{n}, \mathbf{B}^{n}, R^{n}\right) \in \mathbf{J}_{h} \times L_{h} \times \mathbf{X}_{h} \times S_{h}$, such that

$$
\begin{aligned}
&\left(d_{t} \mathbf{U}^{n}, \boldsymbol{\xi}\right)+\frac{1}{\operatorname{Re}}\left(\nabla \mathbf{U}^{n}, \nabla \boldsymbol{\xi}\right)+\left(\left(\mathbf{U}^{n-1} \cdot \nabla\right) \mathbf{U}^{n}, \boldsymbol{\xi}\right)+\frac{1}{2}\left(\left(\operatorname{div} \mathbf{U}^{n-1}\right) \mathbf{U}^{n}, \boldsymbol{\xi}\right) \\
&+ S\left(\mathbf{B}^{n-1} \times \operatorname{curl} \mathbf{B}^{n}, \boldsymbol{\xi}\right)-\left(P^{n}, \operatorname{div} \boldsymbol{\xi}\right)=\left(\mathbf{g}^{n}, \boldsymbol{\xi}\right) \quad \forall \boldsymbol{\xi} \in \mathbf{V}_{h}, \\
&\left(d_{t} \mathbf{B}^{n}, \boldsymbol{\psi}\right)+\frac{1}{\operatorname{Re}_{m}}\left(\operatorname{curl} \mathbf{B}^{n}, \operatorname{curl} \boldsymbol{\psi}\right)-\left(\mathbf{U}^{n} \times \mathbf{B}^{n-1}, \operatorname{curl} \boldsymbol{\psi}\right)-\left(\nabla R^{n}, \boldsymbol{\psi}\right)=0 \quad \forall \boldsymbol{\psi} \in \mathbf{C}_{h} .
\end{aligned}
$$

Remark 3.1. In order to enforce $(1.2)_{2}$, a penalization term $\gamma\left(\operatorname{div} \mathbf{B}^{n}, \operatorname{div} \boldsymbol{\xi}\right)$ for $\gamma>0$ in the context of $\mathbf{W}^{1,2}$-conforming finite elements is added to the left-hand side of (3.14) in [2], in addition to the magnetic pressure $R^{n}$. We refer to [18], Section 6 , and [12], Remark 3.3.1, for a discussion of this regularization strategy; see also Section 4.

Upon choosing $(\xi, \boldsymbol{\psi})=\left(\mathbf{U}^{n}, \mathbf{B}^{n}\right)$, and summing equations (3.1) and (3.2), we obtain the discrete energy identity

$$
\begin{aligned}
\frac{1}{2} d_{t}\left[\left\|\mathbf{U}^{n}\right\|_{\mathbf{L}^{2}}^{2}+S\left\|\mathbf{B}^{n}\right\|_{\mathbf{L}^{2}}^{2}\right]+\frac{k}{2}\left[\left\|d_{t} \mathbf{U}^{n}\right\|_{\mathbf{L}^{2}}^{2}+S\left\|d_{t} \mathbf{B}^{n}\right\|_{\mathbf{L}^{2}}^{2}\right] & \\
& +\frac{1}{\operatorname{Re}}\left\|\nabla \mathbf{U}^{n}\right\|_{\mathbf{L}^{2}}^{2}+\frac{S}{\operatorname{Re}_{m}}\left\|\operatorname{curl} \mathbf{B}^{n}\right\|_{\mathbf{L}^{2}}^{2}=\left(\mathbf{g}^{n}, \mathbf{U}^{n}\right) .
\end{aligned}
$$

This fully discrete scheme is based on the same temporal discretization of (1.1)-(1.3) in [2], where its main motivation is (3.3). Scheme A inherits this property in a fully discrete setting, and is also linear, although coupled; we may use standard arguments for stable mixed finite element discretizations (see e.g. [1], Sect. 4.1, for (3.2)) to conclude unique solvability for Scheme A. 
Lemma 3.1. For every $n \geq 1$, let $\left(\mathbf{U}^{n}, P^{n}, \mathbf{B}^{n}, R^{n}\right) \in \mathbf{J}_{h} \times L_{h} \times \mathbf{X}_{h} \times S_{h}$ be the unique solution of (3.1)-(3.2). There holds

$$
\begin{aligned}
& \frac{1}{2} \max _{1 \leq n \leq N}\left[\left\|\mathbf{U}^{n}\right\|_{\mathbf{L}^{2}}^{2}+S\left\|\mathbf{B}^{n}\right\|_{\mathbf{L}^{2}}^{2}\right]+\frac{k^{2}}{2} \sum_{n=1}^{N}\left[\left\|d_{t} \mathbf{U}^{n}\right\|_{\mathbf{L}^{2}}^{2}+S\left\|d_{t} \mathbf{B}^{n}\right\|_{\mathbf{L}^{2}}^{2}\right] \\
& \quad+k \sum_{n=1}^{N}\left[\frac{1}{\operatorname{Re}}\left\|\nabla \mathbf{U}^{n}\right\|_{\mathbf{L}^{2}}^{2}+\frac{S}{\operatorname{Re}_{m}}\left\|\operatorname{curl} \mathbf{B}^{n}\right\|_{\mathbf{L}^{2}}^{2}\right] \leq \frac{1}{2}\left[\left\|\mathbf{U}^{0}\right\|_{\mathbf{L}^{2}}^{2}+S\left\|\mathbf{B}^{0}\right\|_{\mathbf{L}^{2}}^{2}\right]+k \sum_{n=1}^{N}\left(\mathbf{g}^{n}, \mathbf{U}^{n}\right) .
\end{aligned}
$$

The next assertion controls changes in time of iterates $\left\{\left(\mathbf{U}^{n}, \mathbf{B}^{n}\right)\right\}_{n=1}^{N}$ from Scheme A.

Lemma 3.2. Let $\left\{\left(\mathbf{U}^{n}, \mathbf{B}^{n}\right)\right\}_{n=1}^{N} \subset \mathbf{J}_{h} \times \mathbf{X}_{h}$ solve Scheme A. There exists $r \equiv r\left(\delta_{1}\right)>1$, such that

$$
k \sum_{n=1}^{N}\left\{\left\|d_{t} \mathbf{U}^{n}\right\|_{\left(\mathbf{J} \cap \mathbf{W}^{2,2}\right)^{\prime}}^{r}+\left\|d_{t} \mathbf{B}^{n}\right\|_{\left(\mathbf{X} \cap \mathbf{W}^{2,2}\right)^{\prime}}^{r}\right\} \leq C .
$$

Proof. To validate the first assertion, choose $\boldsymbol{\xi}=\mathbf{Q}_{h}^{0} \mathbf{v} \in \mathbf{J}_{h}$, for every $\mathbf{v} \in \mathbf{J} \cap \mathbf{W}^{2,2}(\Omega)$. Thanks to $\left(d_{t} \mathbf{U}^{n}, \mathbf{v}-\right.$ $\left.\mathbf{Q}_{h}^{0} \mathbf{v}\right)=0,(3.1)$, the continuous embedding $W^{1,2}(\Omega) \hookrightarrow L^{6}(\Omega)$, and $L^{p}$-interpolation, we conclude

$$
\begin{aligned}
\left|\left(d_{t} \mathbf{U}^{n}, \mathbf{v}\right)\right| \leq & C\left[\frac{1}{\operatorname{Re}}\left\|\nabla \mathbf{U}^{n}\right\|_{\mathbf{L}^{2}}+\left\|\mathbf{U}^{n-1}\right\|_{\mathbf{L}^{3}}\left\|\mathbf{U}^{n}\right\|_{\mathbf{L}^{6}}+\left\|\operatorname{div} \mathbf{U}^{n-1}\right\|_{\mathbf{L}^{2}}\left\|\mathbf{U}^{n}\right\|_{\mathbf{L}^{3}}\right. \\
& \left.+\left\|\mathbf{g}^{n}\right\|_{\mathbf{J}^{\prime}}\right]\|\nabla \boldsymbol{\xi}\|_{\mathbf{L}^{2}}+\frac{1}{\operatorname{Re}_{m}} I \\
\leq & C\left[\frac{1}{\operatorname{Re}}\left\|\nabla \mathbf{U}^{n}\right\|_{\mathbf{L}^{2}}+\max _{(i, l) \in\{0,1\}^{2}}\left\|\mathbf{U}^{n-i}\right\|_{\mathbf{L}^{2}}^{1 / 2}\left\|\nabla \mathbf{U}^{n-l}\right\|_{\mathbf{L}^{2}}^{3 / 2}+\left\|\mathbf{g}^{n}\right\|_{\mathbf{J}^{\prime}}\right] \\
& \times\left(\|\mathbf{v}\|_{\mathbf{W}^{1,2}}+h\|\mathbf{v}\|_{\mathbf{W}^{2,2}}\right)+\frac{1}{\operatorname{Re}_{m}} I,
\end{aligned}
$$

where $I:=\left|\left(\mathbf{B}^{n-1} \times \operatorname{curl} \mathbf{B}^{n}, \boldsymbol{\xi}\right)\right|$. We control this term separately. For this purpose, we use the Hodge mapping $\mathbf{H}: \mathbf{X}_{h} \rightarrow \mathbf{X}$, which satisfies (2.14), for some $\sigma \equiv \sigma(\Omega)>0$. We may compute

$$
I \leq\left\|\mathbf{B}^{n-1}-\mathbf{H}\left(\mathbf{B}^{n-1}\right)\right\|_{\mathbf{L}^{2}}\left\|\operatorname{curl} \mathbf{B}^{n}\right\|_{\mathbf{L}^{2}}\|\boldsymbol{\xi}\|_{\mathbf{L}^{\infty}}+\left\|\mathbf{H}\left(\mathbf{B}^{n-1}\right)\right\|_{\mathbf{L}^{3}}\left\|\operatorname{curl} \mathbf{B}^{n}\right\|_{\mathbf{L}^{2}}\|\boldsymbol{\xi}\|_{\mathbf{L}^{6}} .
$$

We use an inverse estimate for the first term, and $L^{p}$-interpolation together with Lemma 2.1(i) for the second. Then, there exists $0<\delta_{2} \equiv \delta_{2}\left(\delta_{1}\right)<1$, such that we find the upper bound

$$
\begin{aligned}
I & \leq C\left[h^{\sigma}\left\|\operatorname{curl} \mathbf{B}^{n-1}\right\|_{\mathbf{L}^{2}}\left\|\operatorname{curl} \mathbf{B}^{n}\right\|_{\mathbf{L}^{2}}+\left\|\mathbf{B}^{n-1}\right\|_{\mathbf{L}^{2}}^{\delta_{2}}\left\|\mathbf{B}^{n-1}\right\|_{\mathbf{H}(\operatorname{curl})}^{1-\delta_{2}}\left\|\operatorname{curl} \mathbf{B}^{n}\right\|_{\mathbf{L}^{2}}\right]\|\boldsymbol{\xi}\|_{\mathbf{L}^{6}} \\
& \leq C\left[\left\|\mathbf{B}^{n-1}\right\|_{\mathbf{L}^{2}}+\left\|\mathbf{B}^{n-1}\right\|_{\mathbf{L}^{2}}^{\delta_{2}}\left\|\operatorname{curl} \mathbf{B}^{n-1}\right\|_{\mathbf{L}^{2}}^{1-\delta_{2}}\right]\left\|\operatorname{curl} \mathbf{B}^{n}\right\|_{\mathbf{L}^{2}}\|\nabla \boldsymbol{\xi}\|_{\mathbf{L}^{2}} .
\end{aligned}
$$

Thanks to the discrete energy law, the first assertion then follows from (3.4), for some existing $r \equiv r\left(\delta_{1}\right)>1$.

To validate the second assertion, choose $\boldsymbol{\psi}=\mathbf{R}_{h}^{0} \mathbf{w} \in \mathbf{X}_{h}$, for every $\mathbf{w} \in \mathbf{X} \cap \mathbf{W}^{2,2}(\Omega)$. Note that $\left(d_{t} \mathbf{B}^{n}, \mathbf{w}-\right.$ $\left.\mathbf{R}_{h}^{0} \mathbf{w}\right)=0$. We use (3.2), Young's inequality, and Lemma 2.1(ii),

$$
\left|\left(d_{t} \mathbf{B}^{n}, \mathbf{w}\right)\right| \leq \frac{1}{\operatorname{Re}_{m}}\left\|\operatorname{curl} \mathbf{B}^{n}\right\|_{\mathbf{L}^{2}}\|\operatorname{curl} \psi\|_{\mathbf{L}^{2}}+I I,
$$


where $I I=\left|\left(\mathbf{U}^{n} \times \mathbf{B}^{n-1}, \operatorname{curl} \psi\right)\right|$. We use similar arguments as for $I$ to obtain

$$
\begin{aligned}
I I & \leq\left\|\mathbf{U}^{n}\right\|_{\mathbf{L}^{\infty}}\left\|\mathbf{B}^{n-1}-\mathbf{H}\left(\mathbf{B}^{n-1}\right)\right\|_{\mathbf{L}^{2}}\|\operatorname{curl} \boldsymbol{\psi}\|_{\mathbf{L}^{2}}+\left\|\mathbf{U}^{n}\right\|_{\mathbf{L}^{6}}\left\|\mathbf{H}\left(\mathbf{B}^{n-1}\right)\right\|_{\mathbf{L}^{3}}\|\operatorname{curl} \boldsymbol{\psi}\|_{\mathbf{L}^{2}} \\
& \leq C\left[h^{\sigma}\left\|\operatorname{curl} \mathbf{B}^{n-1}\right\|_{\mathbf{L}^{2}}+\left\|\mathbf{B}^{n-1}\right\|_{\mathbf{L}^{2}}^{\delta_{2}}\left\|\mathbf{B}^{n-1}\right\|_{\mathbf{H}(\operatorname{curl})}^{1-\delta_{2}}\right]\left\|\mathbf{U}^{n}\right\|_{\mathbf{L}^{6}}\|\operatorname{curl} \boldsymbol{\psi}\|_{\mathbf{L}^{2}}
\end{aligned}
$$

for some $\sigma \equiv \sigma(\Omega)>0$, and $0<\delta_{2} \equiv \delta_{2}\left(\delta_{1}\right)<1$. In order to control $\|$ curl $\mathbf{R}_{h}^{0} \mathbf{w} \|_{\mathbf{L}^{2}}$, we use inverse inequality, and (2.12) to compute

$$
\begin{aligned}
\left\|\operatorname{curl}\left[\mathbf{R}_{h}^{0} \mathbf{w} \pm \mathbf{R}_{h}^{1} \mathbf{w}\right]\right\|_{\mathbf{L}^{2}} \leq & \frac{C}{h}\left\{\left\|\mathbf{w}-\mathbf{R}_{h}^{0} \mathbf{w}\right\|_{\mathbf{L}^{2}}+\left\|\mathbf{w}-\mathbf{R}_{h}^{1} \mathbf{w}\right\|_{\mathbf{L}^{2}}\right\} \\
& +\left\|\operatorname{curl}\left[\mathbf{R}_{h}^{1} \mathbf{w}-\mathbf{w}\right]\right\|_{\mathbf{L}^{2}}+\|\operatorname{curl} \mathbf{w}\|_{\mathbf{L}^{2}} \\
\leq & C\left[(1+h)\|\mathbf{w}\|_{\mathbf{w}^{2,2}}+\|\operatorname{curl} \mathbf{w}\|_{\mathbf{L}^{2}}\right] .
\end{aligned}
$$

In (3.5), we now employ an inverse estimate, and the discrete energy law, to conclude the second assertion of the lemma for some $r \equiv r(\Omega)>1$.

Let $\left\{\left(\mathbf{U}^{n}, P^{n}, \mathbf{B}^{n}, R^{n}\right)\right\}_{n=0}^{N} \subset \mathbf{J}_{h} \times L_{h} \times \mathbf{X}_{h} \times S_{h}$ solve Scheme A; consider corresponding piecewise affine, globally continuous interpolants in time $\{(\mathcal{U}(t, \cdot), \wp(t, \cdot), \mathcal{B}(t, \cdot), R(t, \cdot))\}_{0<t<T}$ as defined in Section 2.5, and depending on $k, h>0$ in particular. By Lemmas 3.1 and 3.2 , for tending $(k, h) \rightarrow 0$, there exists a convergent subsequence of the quadruple, and functions

$$
\mathbf{u} \in L^{\infty}(0, T ; \mathbf{H}) \cap L^{2}(0, T ; \mathbf{J}), \quad \mathbf{b} \in L^{\infty}(0, T ; \mathbf{H}) \cap L^{2}(0, T ; \mathbf{X}),
$$

such that, for some existing $r \equiv r\left(\delta_{1}\right)>1$, and $\delta_{1} \equiv \delta_{1}(\Omega)>0$,

$$
\begin{aligned}
\boldsymbol{U} \stackrel{*}{*} \mathbf{u} & \text { in } L^{\infty}\left(0, T ; \mathbf{L}^{2}\right), \\
\mathcal{U} \rightarrow \mathbf{u} & \text { in } L^{2}\left(0, T ; \mathbf{W}^{1,2}\right) \cap W^{1, r}\left(0, T ;\left[\mathbf{V} \cap \mathbf{W}^{2,2}\right]^{\prime}\right), \\
\mathcal{U}^{ \pm}, \mathcal{U} \rightarrow \mathbf{u} & \text { in } L^{2}\left(0, T ; \mathbf{L}^{q}\right), \quad \forall 1 \leq q<6 \\
\mathcal{B} \stackrel{*}{\rightarrow} \mathbf{b} & \text { in } L^{\infty}\left(0, T ; \mathbf{L}^{2}\right), \\
\mathcal{B} \rightarrow \mathbf{b} & \text { in } L^{2}(0, T ; \mathbf{H}(\operatorname{curl})) \cap W^{1, r}\left(0, T ;\left[\mathbf{X} \cap \mathbf{W}^{2,2}\right]^{\prime}\right), \\
\mathcal{B}^{ \pm}, \mathcal{B} \rightarrow \mathbf{b} & \text { in } L^{2}\left(0, T ; \mathbf{L}^{2}\right) .
\end{aligned}
$$

Weak incompressibility of $\mathbf{u}$ as stated in $(3.6)_{1}$ follows from $\left(\operatorname{div} \mathbf{U}^{n}, \Pi\right)=0$ for all $\Pi \in L_{h}$ : on putting $\Pi=I_{h} \chi \in L_{h}$ for $\chi \in C^{\infty}(\Omega)$, and tending $h \rightarrow 0$ validates the assertion. Also, we use Lemma 2.3 to make sure assertion $(3.6)_{2}$ that $\mathbf{b}$ is divergence-free. We employ Lemmas 2.2 and 2.1(ii) to obtain $(3.7)_{3}$ and (3.7) . $_{6}$ Moreover, from Lemma 3.1 we may conclude that sequences $\{\mathcal{U}\}$ resp. $\left\{\mathcal{U}^{ \pm}\right\}$, as well as $\{\mathcal{B}\}$ resp. $\left\{\mathcal{B}^{ \pm}\right\}$converge to the same limit as $k, h \rightarrow 0$, since for example

$$
\left\|\boldsymbol{U}-\mathcal{U}^{+}\right\|_{L^{2}\left(0, T ; \mathbf{L}^{2}\right)}^{2}=\sum_{n=1}^{N}\left\|\mathbf{U}^{n}-\mathbf{U}^{n-1}\right\|_{\mathbf{L}^{2}}^{2} \int_{t_{n-1}}^{t_{n}}\left(\frac{s-t_{n}}{k}\right)^{2} \mathrm{~d} s=\frac{k^{3}}{3} \sum_{n=1}^{N}\left\|d_{t} \mathbf{U}^{n}\right\|_{\mathbf{L}^{2}}^{2}
$$

which tends to zero for $k \rightarrow 0$.

The following result is that $(\mathbf{u}, \mathbf{b})$ is indeed a weak solution to (1.1)-(1.5).

Theorem 3.1. Suppose that $\Omega \subset \mathbb{R}^{3}$ is a bounded polyhedral domain, and $T:=k N>0$. Let

$$
\left\{\left(\mathbf{U}^{n}, P^{n}, \mathbf{B}^{n}, R^{n}\right)\right\}_{n=1}^{N} \subset \mathbf{J}_{h} \times L_{h} \times \mathbf{X}_{h} \times S_{h}
$$


be solutions to Scheme A. There exist a convergent subsequence, and $(\mathbf{u}, \mathbf{b}) \in\left(L^{\infty}(0, T ; \mathbf{H}) \cap L^{2}(0, T ; \mathbf{J})\right) \times$ $\left(L^{\infty}(0, T ; \mathbf{H}) \cap L^{2}(0, T ; \mathbf{X})\right)$, such that for $k, h \rightarrow 0$, there holds

$$
\mathcal{U} \stackrel{*}{\rightarrow} \mathbf{u} \quad \text { in } L^{\infty}\left(0, T ; \mathbf{L}^{2}\right), \quad \mathcal{B} \stackrel{*}{*} \mathbf{b} \quad \text { in } L^{\infty}\left(0, T ; \mathbf{L}^{2}\right)
$$

Moreover, $(\mathbf{u}, \mathbf{b})$ is a weak solution to $(1.1)-(1.5)$.

Proof. We rewrite (3.1)-(3.2) as follows. For every $t>0$, find $(\mathbf{U}(t, \cdot), \mathbf{B}(t, \cdot)) \in \mathbf{J}_{h} \times \mathbf{X}_{h}$, such that for every $(\xi, \psi) \in \mathbf{J}_{h} \times \mathbf{X}_{h}$

$$
\begin{gathered}
\left(\mathcal{U}_{t}, \boldsymbol{\xi}\right)+\frac{1}{\operatorname{Re}}\left(\nabla \mathcal{U}^{+}, \nabla \boldsymbol{\xi}\right)+\left(\left(\mathcal{U}^{-} \cdot \nabla\right) \mathcal{U}^{+}, \boldsymbol{\xi}\right)+\frac{1}{2}\left(\left(\operatorname{div} \mathcal{U}^{-}\right) \mathcal{U}^{+}, \boldsymbol{\xi}\right)+S\left(\mathcal{B}^{-} \times \operatorname{curl} \mathcal{B}^{+}, \boldsymbol{\xi}\right)=\left(\mathbf{f}^{+}, \boldsymbol{\xi}\right) \\
\left(\mathcal{B}_{t}, \boldsymbol{\psi}\right)+\frac{1}{\operatorname{Re}_{m}}\left(\operatorname{curl} \mathcal{B}^{+}, \operatorname{curl} \boldsymbol{\psi}\right)-\left(\mathcal{U}^{+} \times \mathcal{B}^{-}, \operatorname{curl} \boldsymbol{\psi}\right)=0
\end{gathered}
$$

For every $(\mathbf{v}, \mathbf{w}) \in \mathcal{J} \times \mathcal{W}$, consider $(\boldsymbol{\xi}, \boldsymbol{\psi}) \equiv\left(\mathbf{Q}_{h}^{0} \mathbf{v}, \mathbf{R}_{h}^{0} \mathbf{w}\right) \in \mathbf{J}_{h} \times \mathbf{X}_{h}$. Because of (2.7), (2.12), and inverse inequalities, there holds for $h \rightarrow 0$,

$$
(\xi, \psi) \rightarrow(\mathbf{v}, \mathbf{w}) \quad \text { in } \quad \mathbf{W}^{1, p}(\Omega) \times\left\{\boldsymbol{\varphi} \in \mathbf{H}(\operatorname{curl} ; \Omega): \operatorname{curl} \boldsymbol{\varphi} \in \mathbf{L}^{p}\right\} \quad\left(1 \leq p<\frac{2 d}{d-2}\right)
$$

After extending $(3.8)-(3.9)$ to $(\boldsymbol{\xi}, \boldsymbol{\psi}) \in C_{0}^{\infty}([0, T) ; \mathcal{J}) \times C_{0}^{\infty}([0, T) ; \mathcal{W})$, and integrating by parts in the leading terms of (3.8) and (3.9), by using convergence results (3.7) we may now easily pass to and identify limits for all terms, which validates that $(\mathbf{u}, \mathbf{b})$ satisfies item (iii) of Definition 2.1 as well.

Properties (ii) and (iv) now follow from standard arguments, and Lemma 3.1.

The main advantages of Scheme A are its linear character, the discrete energy identity (3.3), and (subsequence) convergence of iterates towards weak solutions of (1.1)-(1.5) for vanishing discretization parameters in a general context of data. However, solutions $\left(\mathbf{U}^{n}, \mathbf{B}^{n}\right)$ for every $n \geq 1$ are obtained in a coupled manner, and it is therefore tempting to consider segregated schemes; we refer to [15,25], and also [12], Section 3.6, for a discussion of decoupling schemes for the stationary problem. The following fixed-point algorithm allows for a simultaneous computation of new iterates for both, velocity and magnetic field.

Algorithm A. Fix $(k, h)>0$. Let $\left(\mathbf{U}^{0}, \mathbf{B}^{0}\right) \in \mathbf{J}_{h} \times \mathbf{X}_{h}$, and set $n:=1$.

1. Set $\mathbf{U}^{n, 0}:=\mathbf{U}^{n-1}, \mathbf{B}^{n, 0}:=\mathbf{B}^{n-1}$ and $\ell=0$.

2. Compute $\left(\mathbf{U}^{n, \ell} P^{n, \ell}, \mathbf{B}^{n, \ell}, R^{n, \ell}\right) \in \mathbf{J}_{h} \times L_{h} \times \mathbf{X}_{h} \times S_{h}$ such that

$$
\begin{aligned}
& \left(\frac{\mathbf{U}^{n, \ell}-\mathbf{U}^{n-1}}{k}, \boldsymbol{\xi}\right)+\frac{1}{\operatorname{Re}}\left(\nabla \mathbf{U}^{n, \ell}, \nabla \boldsymbol{\xi}\right)+\left(\left(\mathbf{U}^{n-1} \cdot \nabla\right) \mathbf{U}^{n, \ell}, \boldsymbol{\xi}\right)+\frac{1}{2}\left(\left(\operatorname{div} \mathbf{U}^{n-1}\right) \mathbf{U}^{n, \ell}, \boldsymbol{\xi}\right) \\
& \quad+S\left(\mathbf{B}^{n-1} \times \operatorname{curl} \mathbf{B}^{n, \ell-1}, \boldsymbol{\xi}\right)-\left(P^{n, \ell}, \operatorname{div} \boldsymbol{\xi}\right)=\left(\mathbf{g}^{n}, \boldsymbol{\xi}\right) \quad \forall \boldsymbol{\xi} \in \mathbf{V}_{h} \\
& \left(\frac{\mathbf{B}^{n, \ell}-\mathbf{B}^{n-1}}{k}, \boldsymbol{\psi}\right)+\frac{1}{\operatorname{Re}_{m}}\left(\operatorname{curl} \mathbf{B}^{n, \ell}, \operatorname{curl} \boldsymbol{\psi}\right)-\left(\mathbf{U}^{n, \ell-1} \times \mathbf{B}^{n-1}, \operatorname{curl} \boldsymbol{\psi}\right)-\left(\nabla R^{n, \ell}, \boldsymbol{\psi}\right)=0 \quad \forall \boldsymbol{\psi} \in \mathbf{C}_{h} .
\end{aligned}
$$

3. For fixed $\theta>0$ stop if

$$
\left\|\operatorname{curl}\left[\mathbf{B}^{n, \ell-1}-\mathbf{B}^{n, \ell}\right]\right\|_{\mathbf{L}^{3}}+\left\|\mathbf{U}^{n, \ell-1}-\mathbf{U}^{n, \ell}\right\|_{\mathbf{L}^{\infty}} \leq \theta,
$$

set $\mathbf{U}^{n}:=\mathbf{U}^{n, \ell+1}, \mathbf{B}^{n}:=\mathbf{B}^{n, \ell+1}$, and go to Step 1 .

4. Set $\ell:=\ell+1$, and go to Step 2 . 
A difficulty to validate convergence of this fixed point strategy is the lack of a priori estimates for iterates, which are independent of $k, h>0$. This problematic nature is shared by other decoupling strategies, e.g., where the third term in (3.11) is substituted by $-\left(\mathbf{U}^{n, \ell} \times \mathbf{B}^{n-1}, \operatorname{curl} \boldsymbol{\psi}\right)$. The following result gives sufficient conditions to validate convergence of solutions $\left\{\left(\mathbf{U}^{j, \ell}, \mathbf{B}^{j, \ell}\right)\right\}_{j, \ell}$ to weak solutions of (1.1)-(1.5).

Theorem 3.2. Suppose that $\Omega \subset \mathbb{R}^{3}$ is a bounded polyhedral domain, and $T \equiv k N>0$. Let

$$
\left\{\left(\mathbf{U}^{n, \ell}, P^{n, \ell}, \mathbf{B}^{n, \ell}, S^{n, \ell}\right)\right\}_{\substack{1 \leq n \leq N \\ \ell \geq 0}}
$$

be a solution of Algorithm A. There exist $\tilde{C} \equiv \tilde{C}\left(\Omega_{T}, S, \operatorname{Re}, \operatorname{Re}_{m}\right)>0$, and $q<1$ such that $(1 \leq n \leq N)$

$$
\left[\left\|\mathbf{U}^{n, \ell+1}-\mathbf{U}^{n, \ell}\right\|_{\mathbf{L}^{2}}+\left\|\mathbf{B}^{n, \ell+1}-\mathbf{B}^{n, \ell}\right\|_{\mathbf{L}^{2}}\right] \leq q\left[\left\|\mathbf{U}^{n, \ell}-\mathbf{U}^{n, \ell-1}\right\|_{\mathbf{L}^{2}}+\left\|\mathbf{B}^{n, \ell}-\mathbf{B}^{n, \ell-1}\right\|_{\mathbf{L}^{2}}\right] \quad(\ell \geq 1),
$$

provided that $k \leq \tilde{C} h^{4}$. Moreover, the fixed-point algorithm terminates for every $n \geq 1$, and corresponding unique iterates ( $\left.\mathbf{U}^{n, \bar{\ell}_{n}}, \mathbf{B}^{n, \bar{\ell}_{n}}\right) \in \mathbf{J}_{h} \times \mathbf{X}_{h}$ satisfy the perturbed discrete energy law

$$
\begin{aligned}
& \frac{1}{2} \max _{1 \leq n \leq N}\left[\left\|\mathbf{U}^{n}\right\|_{\mathbf{L}^{2}}^{2}+S\left\|\mathbf{B}^{n}\right\|_{\mathbf{L}^{2}}^{2}\right]+\frac{k^{2}}{2} \sum_{n=1}^{N}\left[\left\|d_{t} \mathbf{U}^{n}\right\|_{\mathbf{L}^{2}}^{2}+S\left\|d_{t} \mathbf{B}^{n}\right\|_{\mathbf{L}^{2}}^{2}\right] \\
&+k \sum_{n=1}^{N}\left[\frac{1}{\operatorname{Re}}\left\|\nabla \mathbf{U}^{n}\right\|_{\mathbf{L}^{2}}^{2}+\frac{S}{\operatorname{Re}_{m}}\left\|\operatorname{curl} \mathbf{B}^{n}\right\|_{\mathbf{L}^{2}}^{2}\right] \leq \frac{1}{2}\left[\left\|\mathbf{U}^{0}\right\|_{\mathbf{L}^{2}}^{2}+S\left\|\mathbf{B}^{0}\right\|_{\mathbf{L}^{2}}^{2}\right]+k \sum_{n=1}^{N}\left(\mathbf{g}^{n}, \mathbf{U}^{n}\right) \\
&+C \theta k \sum_{n=1}^{N}\left\|\mathbf{B}^{n-1}\right\|_{\mathbf{L}^{2}}\left[\left\|\nabla \mathbf{U}^{n}\right\|_{\mathbf{L}^{2}}+\left\|\operatorname{curl} \mathbf{B}^{n}\right\|_{\mathbf{L}^{2}}\right]
\end{aligned}
$$

Moreover, $\left(\mathbf{U}^{n, \bar{\ell}_{n}}, \mathbf{B}^{n, \bar{\ell}_{n}}\right) \rightarrow\left(\mathbf{V}^{n}, \mathbf{C}^{n}\right)(\theta \rightarrow 0)$ for every $n \geq 1$, where $\left\{\left(\mathbf{V}^{n}, \mathbf{C}^{n}\right)\right\}_{n=1}^{N} \subset \mathbf{J}_{h} \times \mathbf{X}_{h}$ solves Scheme A, and hence we obtain overall (subsequence) convergence of $\left\{\left(\mathbf{U}^{n, \bar{\ell}_{n}}, \mathbf{B}^{n, \bar{\ell}_{n}}\right)\right\}_{n=1}^{N}$ towards weak solutions of (1.1)-(1.5) for $(k, h, \theta) \rightarrow 0$.

Proof. Step 1: Contraction property. We proceed by induction to derive the contraction property (3.12). Let $\mathbf{E}_{\varphi}^{n, \ell}:=\varphi^{n, \ell}-\varphi^{n, \ell-1}$, for $\varphi \in\{\mathbf{u}, \mathbf{b}, p, r\}$, and suppose $\left\|\mathbf{U}^{n-1}\right\|_{\mathbf{L}^{2}}+\left\|\mathbf{B}^{n-1}\right\|_{\mathbf{L}^{2}} \leq C$ for some $1 \leq n \leq N$. Then

$$
\begin{aligned}
& \frac{1}{k}\left(\mathbf{E}_{\mathbf{u}}^{n, \ell}, \boldsymbol{\xi}\right)+\frac{1}{\operatorname{Re}}\left(\nabla \mathbf{E}_{\mathbf{u}}^{n, \ell}, \nabla \boldsymbol{\xi}\right)+\left(\left(\mathbf{U}^{n-1} \cdot \nabla\right) \mathbf{E}_{\mathbf{u}}^{n, \ell}, \boldsymbol{\xi}\right)+\frac{1}{2}\left(\left(\operatorname{div} \mathbf{U}^{n-1}\right) \mathbf{E}_{\mathbf{u}}^{n, \ell}, \boldsymbol{\xi}\right) \\
&+S\left(\mathbf{B}^{n-1} \times \operatorname{curl} \mathbf{E}_{\mathbf{b}}^{n, \ell-1}, \boldsymbol{\xi}\right)-\left(E_{p}^{n, \ell}, \operatorname{div} \boldsymbol{\xi}\right)=0 \quad \forall \boldsymbol{\xi} \in \mathbf{V}_{h}, \\
& \frac{1}{k}\left(\mathbf{E}_{\mathbf{b}}^{n, \ell}, \boldsymbol{\psi}\right)+\frac{1}{\operatorname{Re}_{m}}\left(\operatorname{curl} \mathbf{E}_{\mathbf{b}}^{n, \ell}, \operatorname{curl} \boldsymbol{\psi}\right)-\left(\mathbf{E}_{\mathbf{u}}^{n, \ell-1} \times \mathbf{B}^{n-1}, \operatorname{curl} \boldsymbol{\psi}\right)-\left(\nabla E_{r}^{n, \ell}, \boldsymbol{\psi}\right)=0 \quad \forall \boldsymbol{\psi} \in \mathbf{C}_{h}
\end{aligned}
$$

Upon choosing $\boldsymbol{\xi}=\mathbf{E}_{\mathbf{u}}^{n, \ell} \in \mathbf{J}_{h}, \psi=\mathbf{E}_{\mathbf{b}}^{n, \ell} \in \mathbf{X}_{h}$ yields

$$
\begin{aligned}
& \frac{1}{k}\left[\left\|\mathbf{E}_{\mathbf{u}}^{n, \ell}\right\|_{\mathbf{L}^{2}}^{2}+\left\|\mathbf{E}_{\mathbf{b}}^{n, \ell}\right\|_{\mathbf{L}^{2}}^{2}\right]+\frac{1}{\operatorname{Re}}\left\|\nabla \mathbf{E}_{\mathbf{u}}^{n, \ell}\right\|_{\mathbf{L}^{2}}^{2}+\frac{1}{\operatorname{Re}_{m}}\left\|\operatorname{curl} \mathbf{E}_{\mathbf{b}}^{n, \ell}\right\|_{\mathbf{L}^{2}}^{2} \leq \\
& S\left\|\mathbf{B}^{n-1}\right\|_{\mathbf{L}^{6}}\left\|\operatorname{curl} \mathbf{E}_{\mathbf{b}}^{n, \ell-1}\right\|_{\mathbf{L}^{2}}\left\|\mathbf{E}_{\mathbf{u}}^{n, \ell}\right\|_{\mathbf{L}^{3}}+\left\|\mathbf{E}_{\mathbf{u}}^{n, \ell-1}\right\|_{\mathbf{L}^{3}}\left\|\mathbf{B}^{n-1}\right\|_{\mathbf{L}^{6}}\left\|\operatorname{curl} \mathbf{E}_{\mathbf{b}}^{n, \ell}\right\|_{\mathbf{L}^{2}} \\
& \leq \frac{1}{2}\left[\frac{1}{\operatorname{Re}_{m}}\left\|\operatorname{curl} \mathbf{E}_{\mathbf{b}}^{n, \ell-1}\right\|_{\mathbf{L}^{2}}^{2}+\frac{1}{\operatorname{Re}}\left\|\nabla \mathbf{E}_{\mathbf{u}}^{n, \ell}\right\|_{\mathbf{L}^{2}}^{2}\right]+C\left\|\mathbf{B}^{n-1}\right\|_{\mathbf{L}^{6}}^{4}\left\|\mathbf{E}_{\mathbf{u}}^{n, \ell}\right\|_{\mathbf{L}^{2}}^{2}
\end{aligned}
$$

where we use interpolation of $L^{3}$ between $L^{2}$ and $L^{6}$. By inverse estimate, $\left\|\mathbf{B}^{n-1}\right\|_{\mathbf{L}^{6}} \leq C h^{-1}\left\|\mathbf{B}^{n-1}\right\|_{\mathbf{L}^{2}}$, which validates (3.12) for some $\tilde{C}>0$, such that $k \leq \tilde{C} h^{4}$. 
Step 2: Overall convergence. As a consequence, Algorithm A terminates, i.e., there exist finite numbers $\left\{\bar{\ell}_{n}\right\}_{n=1}^{N}$ such that the stopping criterion in Step 3. holds for a given thresholding parameter $\theta>0$. In particular, iterates $\left\{\left(\mathbf{U}^{n}, \mathbf{B}^{n}\right)\right\}_{n=1}^{N}$ satisfy

$$
\begin{array}{r}
\left(d_{t} \mathbf{U}^{n}, \boldsymbol{\xi}\right)+\frac{1}{\operatorname{Re}}\left(\nabla \mathbf{U}^{n}, \nabla \boldsymbol{\xi}\right)+\left(\left(\mathbf{U}^{n-1} \cdot \nabla\right) \mathbf{U}^{n}, \boldsymbol{\xi}\right)+\frac{1}{2}\left(\left(\operatorname{div} \mathbf{U}^{n-1}\right) \mathbf{U}^{n}, \boldsymbol{\xi}\right) \\
+S\left(\mathbf{B}^{n-1} \times \operatorname{curl} \mathbf{B}^{n}, \boldsymbol{\xi}\right)-\left(P^{n}, \operatorname{div} \boldsymbol{\xi}\right)=\left(\mathbf{g}^{n}, \boldsymbol{\xi}\right) \\
+S\left(\mathbf{B}^{n-1} \times \operatorname{curl}\left[\mathbf{B}^{n}-\mathbf{B}^{n, \bar{\ell}_{n}}\right], \boldsymbol{\xi}\right) \quad \forall \boldsymbol{\xi} \in \mathbf{V}_{h}, \\
\left(d_{t} \mathbf{B}^{n}, \boldsymbol{\psi}\right)+\frac{1}{\operatorname{Re}_{m}}\left(\operatorname{curl} \mathbf{B}^{n}, \operatorname{curl} \boldsymbol{\psi}\right)-\left(\mathbf{U}^{n} \times \mathbf{B}^{n-1}, \operatorname{curl} \boldsymbol{\psi}\right)-\left(\nabla R^{n}, \boldsymbol{\psi}\right) \\
+\left(\left[\mathbf{U}^{n}-\mathbf{U}^{n, \bar{\ell}_{n}}\right] \times \mathbf{B}^{n-1}, \operatorname{curl} \boldsymbol{\psi}\right)=0 \quad \forall \boldsymbol{\psi} \in \mathbf{C}_{h} .
\end{array}
$$

The estimate (3.13) now follows easily, and (subsequence) convergence of iterates $\left\{\left(\mathbf{U}^{n, \bar{\ell}_{n}}, \mathbf{B}^{n, \bar{\ell}_{n}}\right)\right\}_{n=1}^{N}$ towards weak solutions of (1.1)-(1.5) is a consequence of Theorem 3.1.

The strong coupling of iterates in Scheme A is manifested by the restrictive constraint $k \leq \tilde{C} h^{4}$ (and $k \leq C h^{2}$ in a $2 \mathrm{D}$ setting) which is sufficient in the decoupling Algorithm A to avoid possible spurious energy transfer between hydrodynamical and electromagnetic part of the overall system, and validate convergence of iterates towards weak solutions of (1.1)-(1.5). We remark that no mesh-constraint applies in case $\left\|\mathbf{B}^{n-1}\right\|_{\mathbf{L}^{6}} \leq C$ would be available; cf. also Section 4, where convergent discretizations of (1.1)-(1.5) for more regular solutions of (1.1)-(1.5) are discussed.

The following scheme is a variant of Scheme A, and decouples hydrodynamical and electromagnetic part. As will be clear from the following, solutions again satisfy a slightly perturbed energy law, provided discretization parameters satisfy $k \leq \tilde{C} h^{3}$ (and $k \leq \tilde{C} h^{2}$ in a $2 \mathrm{D}$ setting), which is made possible by the dissipative character of the implicit Euler method.

Scheme B. Let $n \geq 1$. Find $\left(\mathbf{U}^{n}, P^{n}, \mathbf{B}^{n}, R^{n}\right) \in \mathbf{J}_{h} \times L_{h} \times \mathbf{X}_{h} \times S_{h}$, such that

$$
\begin{aligned}
&\left(d_{t} \mathbf{U}^{n}, \boldsymbol{\xi}\right)+\frac{1}{\operatorname{Re}}\left(\nabla \mathbf{U}^{n}, \nabla \boldsymbol{\xi}\right)+\left(\left(\mathbf{U}^{n-1} \cdot \nabla\right) \mathbf{U}^{n}, \boldsymbol{\xi}\right)+\frac{1}{2}\left(\left[\operatorname{div} \mathbf{U}^{n-1}\right] \mathbf{U}^{n}, \boldsymbol{\xi}\right) \\
&+S\left(\mathbf{B}^{n-1} \times \operatorname{curl} \mathbf{B}^{n-1}, \boldsymbol{\xi}\right)-\left(P^{n}, \operatorname{div} \boldsymbol{\xi}\right)=\left(\mathbf{g}^{n}, \boldsymbol{\xi}\right) \quad \forall \boldsymbol{\xi} \in \mathbf{V}_{h} \\
&\left(d_{t} \mathbf{B}^{n}, \boldsymbol{\psi}\right)+\frac{1}{\operatorname{Re}_{m}}\left(\operatorname{curl} \mathbf{B}^{n}, \operatorname{curl} \boldsymbol{\psi}\right)-\left(\mathbf{U}^{n-1} \times \mathbf{B}^{n-1}, \operatorname{curl} \boldsymbol{\psi}\right)-\left(\nabla R^{n}, \boldsymbol{\psi}\right)=0 \quad \forall \boldsymbol{\psi} \in \mathbf{C}_{h}
\end{aligned}
$$

In order to obtain the discrete energy identity (3.3) for Scheme B, we choose $(\boldsymbol{\xi}, \boldsymbol{\psi})=\left(\mathbf{U}^{n}, \mathbf{B}^{n}\right)$. We obtain for the coupling terms

$$
\begin{aligned}
\left(\mathbf{B}^{n-1} \times \operatorname{curl} \mathbf{B}^{n-1}, \mathbf{U}^{n}\right)-\left(\mathbf{U}^{n-1} \times \mathbf{B}^{n-1}, \operatorname{curl} \mathbf{B}^{n}\right)=\left(\mathbf{B}^{n-1} \times \operatorname{curl} \mathbf{B}^{n-1}, \mathbf{U}^{n-1}\right) \\
\quad-\left(\mathbf{U}^{n-1} \times \mathbf{B}^{n-1}, \operatorname{curl} \mathbf{B}^{n-1}\right)-k\left(\mathbf{B}^{n-1} \times \operatorname{curl} \mathbf{B}^{n-1}, d_{t} \mathbf{U}^{n}\right)+k\left(\mathbf{U}^{n-1} \times \mathbf{B}^{n-1}, \operatorname{curl} d_{t} \mathbf{B}^{n}\right) \\
\geq-C k\left[\left\|\mathbf{B}^{n-1}\right\|_{\mathbf{L}^{\infty}}\left\|\operatorname{curl} \mathbf{B}^{n-1}\right\|_{\mathbf{L}^{2}}\left\|d_{t} \mathbf{U}^{n}\right\|_{\mathbf{L}^{2}}+\left\|\mathbf{U}^{n-1}\right\|_{\mathbf{L}^{6}}\left\|\mathbf{B}^{n-1}\right\|_{\mathbf{L}^{2}}\left\|\operatorname{curl} d_{t} \mathbf{B}^{n}\right\|_{\mathbf{L}^{3}}\right] \\
\geq-C k h^{-3 / 2}\left\|\mathbf{B}^{n-1}\right\|_{\mathbf{L}^{2}}\left[\left\|\operatorname{curl} \mathbf{B}^{n-1}\right\|_{\mathbf{L}^{2}}\left\|d_{t} \mathbf{U}^{n}\right\|_{\mathbf{L}^{2}}+\left\|\nabla \mathbf{U}^{n-1}\right\|_{\mathbf{L}^{2}}\left\|d_{t} \mathbf{B}^{n}\right\|_{\mathbf{L}^{2}}\right] \\
\geq-\frac{k}{4}\left[\left\|d_{t} \mathbf{U}^{n}\right\|_{\mathbf{L}^{2}}^{2}+\frac{1}{\operatorname{Re}_{m}}\left\|d_{t} \mathbf{B}^{n}\right\|_{\mathbf{L}^{2}}^{2}\right]+C k h^{-3}\left\|\mathbf{B}^{n-1}\right\|_{\mathbf{L}^{2}}^{2}\left[\left\|\operatorname{curl} \mathbf{B}^{n-1}\right\|_{\mathbf{L}^{2}}^{2}+\left\|\nabla \mathbf{U}^{n-1}\right\|_{\mathbf{L}^{2}}^{2}\right]
\end{aligned}
$$


by inverse estimates, and Young's inequality. We may now proceed by induction to conclude that there exists $\tilde{C} \equiv \tilde{C}\left(\Omega_{T}\right)>0$, such that for sufficiently small $k \leq \tilde{C} h^{3}$ there holds

$$
\begin{aligned}
\frac{1}{2} \max _{1 \leq n \leq N}\left[\left\|\mathbf{U}^{n}\right\|_{\mathbf{L}^{2}}^{2}+S\left\|\mathbf{B}^{n}\right\|_{\mathbf{L}^{2}}^{2}\right]+\frac{k^{2}}{4} \sum_{n=1}^{N}\left[\left\|d_{t} \mathbf{U}^{n}\right\|_{\mathbf{L}^{2}}^{2}+S\left\|d_{t} \mathbf{B}^{n}\right\|_{\mathbf{L}^{2}}^{2}\right] \\
+\frac{k}{2} \sum_{n=1}^{N}\left[\frac{1}{\operatorname{Re}}\left\|\nabla \mathbf{U}^{n}\right\|_{\mathbf{L}^{2}}^{2}+\frac{S}{\operatorname{Re} m}\left\|\operatorname{curl} \mathbf{B}^{n}\right\|_{\mathbf{L}^{2}}^{2}\right] \leq \frac{1}{2}\left[\left\|\mathbf{U}^{0}\right\|_{\mathbf{L}^{2}}^{2}+S\left\|\mathbf{B}^{0}\right\|_{\mathbf{L}^{2}}^{2}\right]+k \sum_{n=1}^{N}\left(\mathbf{g}^{n}, \mathbf{U}^{n}\right)
\end{aligned}
$$

Once this estimate is available, we obtain the assertion of Lemma 3.2 as well, and the convergence results in (3.7). By following the steps in Theorem 3.1, we find that corresponding limits in (3.7) are a weak solution to (1.1)-(1.5), provided $k \leq \tilde{C} h^{3}$ for some $\tilde{C} \equiv \tilde{C}\left(\Omega_{T}\right)>0$ is respected throughout the limiting process.

Theorem 3.3. Let $\Omega \subset \mathbb{R}^{3}$ be a bounded polyhedral domain, and $T:=k N>0$. Suppose that $k \leq \tilde{C} h^{3}$ is satisfied, for some $\tilde{C} \equiv \tilde{C}\left(\Omega_{T}\right)>0$. Let

$$
\left\{\left(\mathbf{U}^{n}, P^{n}, \mathbf{B}^{n}, R^{n}\right)\right\}_{n=0}^{N} \subset \mathbf{J}_{h} \times L_{h} \times \mathbf{X}_{h} \times S_{h}
$$

be a solution to Scheme B. There exist a convergent subsequence, and $(\mathbf{u}, \mathbf{b}) \in\left(L^{\infty}(0, T ; \mathbf{H}) \cap L^{2}(0, T ; \mathbf{J})\right) \times$ $\left(L^{\infty}(0, T ; \mathbf{H}) \cap L^{2}(0, T ; \mathbf{X})\right)$, such that for $k, h \rightarrow 0$, there holds

$$
\mathcal{U} \stackrel{*}{\rightarrow} \mathbf{u} \quad \text { in } L^{\infty}\left(0, T ; \mathbf{L}^{2}\right), \quad \mathcal{B} \stackrel{*}{\rightarrow} \mathbf{b} \quad \text { in } L^{\infty}\left(0, T ; \mathbf{L}^{2}\right) .
$$

Moreover, $(\mathbf{u}, \mathbf{b})$ is a weak solution to (1.1)-(1.5).

Remark 3.2. (1) In [25], a stable finite element discretization of the stationary incompressible MHD system is proposed, which corresponds to the one employed in Schemes A and B. It is shown in [25], Corollary 4.1, that approximate solutions converge to weak solutions of the stationary MHD system for $h \rightarrow 0$, on general bounded polyhedral domains $\Omega \subset \mathbb{R}^{3}$.

(2) In [15], Section 7, [25], Section 3.4, and [12], Section 3.6, different iterative decoupling strategies for the stationary case to solve the resulting algebraic problem are reviewed: as is pointed out, decoupling strategies, as well as Newton's method often only converge for initial guesses that are sufficiently close to the exact solution, and depends on the value of $\operatorname{Re}>0$, in particular.

(3) Sufficient (restrictive) mesh constraints $F(k, h) \geq 0$ are given to verify a contraction property (Algorithm A), or establish an inductive argument for Scheme B to validate (3.17), which result from using different inverse estimates. In [3], computational experiments compare the proposed schemes (in a related context), study necessary mesh-constraints for practical convergence, as well as compare edge elements to $W^{1,2}$-conforming elements, which by theory require less restrictive inverse estimates.

\section{An efficient SPLitTing SCHeme}

So far, convergence of the coupled Scheme A (unconditional), the decoupling Algorithm A (conditional), and the decoupled Scheme B (conditional) towards weak solutions of (1.1)-(1.5) has been shown. The main tool for this purpose is a discrete energy inequality, which allows to construct weak solutions in a limiting process for vanishing discretization and thresholding parameters. In this section, we take another stand-point by assuming that strong solutions of (1.1)-(1.5) exist, and approximating them by efficient schemes. We propose an optimally convergent scheme which is much simpler if compared to Schemes A and B, and uses nodal elements instead of Nedelec elements. As a result of these simplifications, no discrete energy law is available any more - which 
leaves unclear whether computed iterates converge in a general context where only weak solutions of (1.1)-(1.5) are known to exist.

We make the following assumptions below (see Sect. 2.3 for used notation):

(A1) (regularity of the domain) Let $\Omega \subset \mathbb{R}^{3}$ be a bounded domain, such that unique solutions $(\mathbf{u}, \mathbf{b}) \in \mathbf{J} \times \mathbf{X}$ of $\mathcal{A}_{1} \mathbf{u}=\mathbf{f} \in \mathbf{L}^{2}(\Omega)$ resp. $\mathcal{A}_{2} \mathbf{b}=\mathbf{g} \in \mathbf{L}^{2}(\Omega)$ satisfy $(\mathbf{u}, \mathbf{b}) \in\left[\mathbf{W}^{2,2}(\Omega)\right]^{2}$, and

$$
\|\mathbf{u}\|_{\mathbf{W}^{2,2}} \leq C\|\mathbf{f}\|_{\mathbf{L}^{2}} \quad \text { resp. } \quad\|\mathbf{b}\|_{\mathbf{W}^{2,2}} \leq C\|\mathbf{g}\|_{\mathbf{L}^{2}} .
$$

(A2) (regularity of the data) Let $\left(\mathbf{u}_{0}, \mathbf{b}_{0}\right) \in\left[\mathbf{W}^{2,2}(\Omega)\right]^{2}$, and $\mathbf{g} \in W^{1,2}\left(0, T ; \mathbf{L}^{2}(\Omega)\right)$. Moreover, for every given $T>0$, the tuple $(\mathbf{u}, \mathbf{b}) \in\left[L^{\infty}\left(0, T ; \mathbf{W}^{2,2}\right)\right]^{2}$ is strong solution to (1.1)-(1.5).

We remark that additional regularity of $\Omega \subset \mathbb{R}^{3}$ is needed to make sure that (A1) holds in a general setting; see the discussion in Section 2.3.

The following Scheme $\mathrm{C}$ differs from the above Scheme A in the following aspects:

(i) finite element spaces for velocity field resp. pressure are

$$
\mathbf{V}_{h}=\left\{\boldsymbol{\xi} \in \mathbf{C}_{0}(\bar{\Omega}): \boldsymbol{\xi} \in P_{1}\left(K, \mathbb{R}^{3}\right) \quad \forall K \in \mathcal{T}_{h}\right\}, \quad \tilde{L}_{h}=\left\{\chi \in C(\bar{\Omega}): \chi \in P_{1}(K) \quad \forall K \in \mathcal{T}_{h}\right\},
$$

which now both use piecewise affine, globally continuous functions;

(ii) a consistent regularization term $-\gamma \nabla \operatorname{div} \mathbf{B}$ on the left-hand side of (1.3) is added;

(iii) $\mathbf{W}^{1,2}(\Omega)$-conforming finite elements to approximate the magnetic field $\mathbf{b}: \Omega_{T} \rightarrow \mathbb{R}^{3}$ are used, where

$$
\tilde{\mathbf{C}}_{h}=\left\{\boldsymbol{\psi} \in \mathbf{C}(\bar{\Omega}): \psi \in P_{1}\left(\Omega, \mathbb{R}^{3}\right) \forall K \in \mathcal{T}_{h}\right\} \subset \mathbf{H}_{0}(\operatorname{div} ; \Omega) \cap \mathbf{W}^{1,2}(\Omega)
$$

now ensembles piecewise affine, globally continuous functions;

(iv) the hydrodynamical and electromagnetic parts are decoupled;

(v) a modification of Chorin's projection method to solve the incompressible Navier-Stokes equation [8,22,27] is used.

The computation of iterates for velocity field, pressure, and magnetic field are fully decoupled in the following scheme.

Scheme C. Let $n \geq 1$. 1. For given $\left(\mathbf{U}^{n-1}, \mathbf{B}^{n-1}\right) \in \mathbf{J}_{h} \times \tilde{\mathbf{C}}_{h}$ let $\tilde{\mathbf{U}}^{n} \in \mathbf{V}_{h}$ be a solution of

$$
\begin{aligned}
\frac{1}{k}\left(\tilde{\mathbf{U}}^{n}-\mathbf{U}^{n-1}, \boldsymbol{\xi}\right)+\frac{1}{\operatorname{Re}}\left(\nabla \tilde{\mathbf{U}}^{n}, \nabla \boldsymbol{\xi}\right)+\left(\left(\mathbf{U}^{n-1} \cdot \nabla\right) \tilde{\mathbf{U}}^{n}, \boldsymbol{\xi}\right)+ & \frac{1}{2}\left(\left(\operatorname{div} \mathbf{U}^{n-1}\right) \tilde{\mathbf{U}}^{n}, \boldsymbol{\xi}\right) \\
& +S\left(\mathbf{B}^{n-1} \times \operatorname{curl} \mathbf{B}^{n-1}, \boldsymbol{\xi}\right)=\left(\mathbf{g}^{n}, \boldsymbol{\xi}\right) \quad \forall \boldsymbol{\xi} \in \mathbf{V}_{h} .
\end{aligned}
$$

2. For given $\tilde{\mathbf{U}}^{n} \in \mathbf{V}_{h}$ compute $\left(\mathbf{U}^{n}, P^{n}\right) \in \mathbf{J}_{h} \times \tilde{L}_{h}$ from

$$
\begin{aligned}
\left(\nabla P^{n}, \nabla \chi\right) & =-\frac{1}{k}\left(\operatorname{div} \tilde{\mathbf{U}}^{n}, \chi\right) \quad \forall \chi \in \tilde{L}_{h}, \\
\left(\mathbf{U}^{n}, \boldsymbol{\xi}\right) & =\left(\tilde{\mathbf{U}}^{n}, \boldsymbol{\xi}\right)+k\left(p^{n}, \operatorname{div} \boldsymbol{\xi}\right)=0 \quad \forall \boldsymbol{\xi} \in \mathbf{V}_{h} .
\end{aligned}
$$

3. Let $\mathbf{B}^{n} \in \tilde{\mathbf{C}}_{h}$ solve for all $\boldsymbol{\psi} \in \tilde{\mathbf{C}}_{h}$ that $(\gamma>0)$

$$
\left(d_{t} \mathbf{B}^{n}, \boldsymbol{\psi}\right)+\frac{1}{\operatorname{Re}_{m}}\left(\operatorname{curl} \mathbf{B}^{n}, \operatorname{curl} \boldsymbol{\psi}\right)+\gamma\left(\operatorname{div} \mathbf{B}^{n}, \operatorname{div} \boldsymbol{\psi}\right)-\left(\tilde{\mathbf{U}}^{n-1} \times \mathbf{B}^{n-1}, \operatorname{curl} \boldsymbol{\psi}\right)=0
$$

The first step allows for a component-wise computation of new iterates of the velocity field. In Step 2, the new pressure iterate solves a Poisson equation, and the computation of the new discretely solenoidal velocity 
field $\mathbf{U}^{n}$ only requires to invert a mass matrix. The third step couples with updates of the velocity field in an explicit manner. All steps require to solve linear problems. For every $n \geq 1$, a reformulation of this scheme for iterates $\left(\tilde{\mathbf{U}}^{n}, P^{n}, \mathbf{B}^{n}\right)$ is

$$
\begin{aligned}
\left(d_{t} \tilde{\mathbf{U}}^{n}, \boldsymbol{\xi}\right)+\frac{1}{\operatorname{Re}}\left(\nabla \tilde{\mathbf{U}}^{n}, \nabla \boldsymbol{\xi}\right)+\left(\left(\tilde{\mathbf{U}}^{n-1} \cdot \nabla\right) \tilde{\mathbf{U}}^{n}, \boldsymbol{\xi}\right)+ & \frac{1}{2}\left(\left(\operatorname{div} \tilde{\mathbf{U}}^{n-1}\right) \tilde{\mathbf{U}}^{n}, \boldsymbol{\xi}\right) \\
& +S\left(\mathbf{B}^{n-1} \times \operatorname{curl} \mathbf{B}^{n-1}, \boldsymbol{\xi}\right)+\left(\nabla P^{n-1}, \boldsymbol{\xi}\right)=\left(\mathbf{g}^{n}, \boldsymbol{\xi}\right) \quad \forall \boldsymbol{\xi} \in \mathbf{V}_{h}, \\
\left(\operatorname{div} \tilde{\mathbf{U}}^{n}, \chi\right)+k\left(\nabla P^{n}, \nabla \chi\right)=0 \quad \forall \chi \in \tilde{L}_{h}, & \forall\left(\operatorname{div} \mathbf{B}^{n}, \operatorname{div} \boldsymbol{\psi}\right)-\left(\tilde{\mathbf{U}}^{n-1} \times \mathbf{B}^{n-1}, \operatorname{curl} \boldsymbol{\psi}\right)=0 \quad \forall \boldsymbol{\psi} \in \tilde{\mathbf{C}}_{h} .
\end{aligned}
$$

Equations (4.1)-(4.3) show space-time discretization, semi-explicit treatment of several nonlinear terms, as well as a perturbation of the (discrete) incompressibility constraint in (4.1), and an explicit treatment of the related Lagrange multiplier in (4.1). The 'quasi-compressibility' equation (4.2) implies that the finite element discretization based on the $P 1 / P 1$-element for the hydrodynamic part is stable, once $k \geq C h^{2}$ is satisfied, cf. [19,22]; LBB-stable pairings with proper approximation properties are suitable as well, and $k \geq C h^{2}$ is not needed there. Unfortunately, the characterization (4.1)-(4.3) of iterates computed from Scheme C does not allow for a discrete energy estimate. In fact, the following theorem validates convergence of iterates $\left\{\left(\tilde{\mathbf{U}}^{n}, \mathbf{B}^{n}\right)\right\}_{n=1}^{N}$ of optimal order in terms of discretization parameters $k, h>0$, provided strong solutions to (1.1)-(1.5) exist.

Theorem 4.1. Let $T=k N>0$, and $\left\{\left(\tilde{\mathbf{U}}^{n}, P^{n}, \mathbf{B}^{n}\right)\right\}_{n=1}^{N}$ solves Scheme C. Suppose that (A1), (A2) are valid, as well as

$$
\left[\left\|\mathbf{u}^{0}-\mathbf{U}^{0}\right\|_{\mathbf{L}^{2}}+\left\|\mathbf{b}^{0}-\mathbf{B}^{0}\right\|_{\mathbf{L}^{2}}\right]+(\sqrt{k}+h)\left[\left\|\mathbf{u}^{0}-\mathbf{U}^{0}\right\|_{\mathbf{W}^{1,2}}+\left\|\mathbf{b}^{0}-\mathbf{B}^{0}\right\|_{\mathbf{W}^{1,2}}\right] \leq C\left(k+h^{2}\right) .
$$

For sufficiently small time-steps $k \leq k_{0}(T)$, and $h \leq h_{0}(T)$, there exists $C \equiv C\left(\Omega_{T}, S, \operatorname{Re}, \operatorname{Re}_{m}\right)>0$, such that $\left(\tau^{n}=\min \left\{1, t_{n}\right\}\right)$

$$
\begin{gathered}
\max _{1 \leq n \leq N}\left[\left\|\mathbf{u}\left(t_{n}, \cdot\right)-\tilde{\mathbf{U}}^{n}\right\|_{\mathbf{L}^{2}}+\tau^{n}\left\|p\left(t_{n}, \cdot\right)-P^{n}\right\|_{W^{-1,2}}+\left\|\mathbf{b}\left(t_{n}, \cdot\right)-\mathbf{B}^{n}\right\|_{\mathbf{L}^{2}}\right] \leq C\left(k+h^{2}\right), \\
\max _{1 \leq n \leq N} \sqrt{\tau_{n}}\left\|p\left(t_{n}, \cdot\right)-P^{n}\right\|_{L_{0}^{2}}+\left(k \sum_{n=1}^{N}\left\|\nabla \mathbf{P}_{\mathbf{W}^{1,2}}\left[\mathbf{u}\left(t_{n}, \cdot\right)-\tilde{\mathbf{U}}^{n}\right]\right\|_{\mathbf{L}^{2}}^{2}\right)^{1 / 2} \\
+\left(k \sum_{n=1}^{N}\left\|\nabla \mathbf{P}_{\mathbf{W}^{1,2}}\left[\mathbf{b}\left(t_{n}, \cdot\right)-\tilde{\mathbf{B}}^{n}\right]\right\|_{\mathbf{L}^{2}}^{2}\right)^{1 / 2} \leq C(\sqrt{k}+h) .
\end{gathered}
$$

The proof is split into several steps, each of which accounts for approximation effects due to (semi-implicit) time-discretization, perturbation of the incompressibility constraint, and spatial discretization. Being provided with a strong solution of $(1.1)-(1.5)$, where $\left(\mathbf{u}_{0}, \mathbf{b}_{0}\right) \in\left[\mathbf{W}^{2,2}(\Omega)\right]^{2}$ and $\mathbf{g} \in W^{1,2}\left(0, T ; \mathbf{L}^{2}(\Omega)\right)$, we may easily verify the following bounds,

$$
\begin{aligned}
\|\mathbf{u}\|_{L^{\infty}\left(0, T ; \mathbf{W}^{2,2}\right) \cap W^{1, \infty}\left(0, T ; \mathbf{L}^{2}\right) \cap W^{1,2}\left(0, T ; \mathbf{W}^{1,2}\right) \cap W^{2,2}\left(0, T ; \mathbf{J}^{\prime}\right)}+\|p\|_{L^{\infty}\left(0, T ; W^{1,2}\right) \cap W^{1,2}\left(0, T ; L_{0}^{2}\right)} \\
+\|\mathbf{b}\|_{L^{\infty}\left(0, T ; \mathbf{W}^{2,2}\right) \cap W^{1, \infty}\left(0, T ; \mathbf{L}^{2}\right) \cap W^{1,2}\left(0, T ; \mathbf{W}^{1,2}\right) \cap W^{2,2}\left(0, T ; \mathbf{X}^{\prime}\right)} \leq C .
\end{aligned}
$$

In order to verify Theorem 4.1, we start with an error analysis which accounts for temporal discretization effects. For every $1 \leq n \leq N$, let $\left(\mathbf{u}^{n}, p^{n}, \mathbf{b}^{n}\right) \in \mathbf{W}_{0}^{1,2}(\Omega) \times\left[W^{1,2}(\Omega) \cap L_{0}^{2}(\Omega)\right] \times\left[\mathbf{W}^{1,2}(\Omega) \cap \mathbf{H}_{0}(\operatorname{div} ; \Omega)\right]$ be 
the solution of

$$
\begin{array}{r}
\left(d_{t} \mathbf{u}^{n}, \boldsymbol{\xi}\right)+\frac{1}{\operatorname{Re}}\left(\nabla \mathbf{u}^{n}, \nabla \boldsymbol{\xi}\right)+\left(\left(\mathbf{u}^{n-1} \cdot \nabla\right) \mathbf{u}^{n}, \boldsymbol{\xi}\right)+ \\
+S\left(\mathbf{b}^{n-1} \times \operatorname{curl}^{n-1}, \boldsymbol{\xi}\right)+\left(\nabla p^{n-1}, \boldsymbol{\xi}\right)=\left(\mathbf{g}^{n}, \boldsymbol{\xi}\right) \quad \forall \boldsymbol{\xi} \in \mathbf{W}_{0}^{1,2}(\Omega), \\
\left(\operatorname{div} \mathbf{u}^{n-1}, \chi\right)+k\left(\nabla p^{n}, \nabla \chi\right)=0 \quad \forall \chi \in W^{1,2}(\Omega) \cap L_{0}^{2}(\Omega), \\
\left(d_{t} \mathbf{b}^{n}, \boldsymbol{\psi}\right)+\frac{1}{\operatorname{Re}_{m}}\left(\operatorname{curl} \mathbf{b}^{n}, \operatorname{curl} \boldsymbol{\psi}\right)+\gamma\left(\operatorname{div} \mathbf{b}^{n}, \operatorname{div} \boldsymbol{\psi}\right) \\
-\left(\mathbf{u}^{n-1} \times \mathbf{b}^{n-1}, \operatorname{curl} \boldsymbol{\psi}\right)=0 \quad \forall \boldsymbol{\psi} \in \mathbf{H}_{0}(\operatorname{div} ; \Omega) \cap \mathbf{W}^{1,2}(\Omega) .
\end{array}
$$

The following analysis of (4.6)-(4.8) is split into several steps, each of which addresses different approximation effects due to implicit time discretization, decoupling of hydrodynamic and and electromagnetic part, as well as splitting the Stokes operator to independently compute velocity field and pressure, and an associated perturbation of the incompressibility constraint for the velocity field.

Lemma 4.1. Let $T=k N>0$, and $\left\{\left(\tilde{\mathbf{u}}^{n}, p^{n}, \mathbf{b}^{n}\right)\right\}_{n=1}^{N}$ solves (4.6)-(4.8). Suppose that (A1), (A2) are valid, as well as (4.4), with $h=0$. For sufficiently small time-steps $k \leq k_{0}\left(\Omega_{T}\right)$, there exists $C \equiv C\left(\Omega_{T}, S, \operatorname{Re}, \operatorname{Re}_{m}\right)>0$, such that

(a)

$$
\begin{aligned}
\max _{1 \leq n \leq N}\left[\left\|\mathbf{u}\left(t_{n}, \cdot\right)-\tilde{\mathbf{u}}^{n}\right\|_{\mathbf{L}^{2}}+\tau^{n}\left\|p\left(t_{n}, \cdot\right)-p^{n}\right\|_{W^{-1,2}}+\left\|\mathbf{b}\left(t_{n}, \cdot\right)-\mathbf{b}^{n}\right\|_{\mathbf{L}^{2}}\right] \leq C k \\
\max _{1 \leq n \leq N} \sqrt{\tau_{n} k}\left\|p\left(t_{n}, \cdot\right)-p^{n}\right\|_{L_{0}^{2}}+\left(k \sum_{n=1}^{N}\left\|\mathbf{u}\left(t_{n}, \cdot\right)-\tilde{\mathbf{u}}^{n}\right\|_{\mathbf{W}^{1,2}}^{2}\right)^{1 / 2} \\
+\left(k \sum_{n=1}^{N}\left\|\mathbf{b}\left(t_{n}, \cdot\right)-\mathbf{b}^{n}\right\|_{\mathbf{W}^{1,2}}^{2}\right)^{1 / 2} \leq C k
\end{aligned}
$$

In below, we say that the triple $\left\{\left(\boldsymbol{\xi}^{n}, \boldsymbol{\psi}^{n}, \chi^{n}\right)\right\}_{n=1}^{N} \subset\left[\mathbf{L}^{2}\right]^{2} \times L_{0}^{2}$ satisfies property:

(P1) if the following bounds hold uniformly in $k>0$,

$$
\begin{aligned}
\max _{1 \leq n \leq N}\left\{\left\|\boldsymbol{\xi}^{n}\right\|_{\mathbf{W}^{2,2}}+\left\|\boldsymbol{\psi}^{n}\right\|_{\mathbf{W}^{2,2}}+\left\|\chi^{n}\right\|_{W^{1,2} \cap L_{0}^{2}}+\left\|d_{t} \boldsymbol{\xi}^{n}\right\|_{\mathbf{L}^{2}}+\left\|d_{t} \boldsymbol{\psi}^{n}\right\|_{\mathbf{L}^{2}}\right\} & \\
& +\left(k \sum_{1 \leq n \leq N}\left[\left\|d_{t} \boldsymbol{\xi}^{n}\right\|_{\mathbf{W}^{1,2}}^{2}+\left\|d_{t} \boldsymbol{\psi}^{n}\right\|_{\mathbf{W}^{1,2}}^{2}+\left\|d_{t} \chi^{n}\right\|_{L_{0}^{2}}^{2}\right]\right)^{1 / 2} \leq C ;
\end{aligned}
$$

(P2) if the following error estimates are satisfied, for $\tau^{n}:=\min \left\{t_{n}, 1\right\}$,

$$
\begin{aligned}
\max _{1 \leq n \leq N}\left\{\left\|\mathbf{u}\left(t_{n}, \cdot\right)-\boldsymbol{\xi}^{n}\right\|_{\mathbf{L}^{2}}+\left\|\mathbf{b}\left(t_{n}, \cdot\right)-\boldsymbol{\psi}^{n}\right\|_{\mathbf{L}^{2}}\right. & \left.+\tau^{n}\left\|p\left(t_{n}\right)-\chi^{n}\right\|_{W^{-1,2}}+\sqrt{\tau^{n} k}\left\|p\left(t_{n}, \cdot\right)-\chi^{n}\right\|_{L_{0}^{2}}\right\} \\
& +\sqrt{k}\left(k \sum_{n=1}^{N}\left[\left\|\mathbf{u}\left(t_{n}, \cdot\right)-\boldsymbol{\xi}^{n}\right\|_{\mathbf{W}^{1,2}}+\left\|\mathbf{b}\left(t_{n}, \cdot\right)-\boldsymbol{\psi}^{n}\right\|_{\mathbf{W}^{1,2}}\right]\right)^{1 / 2} \leq C k
\end{aligned}
$$


Proof of Lemma 4.1. Step 1: Implicit discretization of (1.1)-(1.5). For every $1 \leq n \leq N$, let $\left(\mathbf{u}_{A}^{n}, p_{A}^{n}, \mathbf{b}_{A}^{n}\right) \in$ $\mathbf{J} \times\left[W^{1,2}(\Omega) \cap L_{0}^{2}(\Omega)\right] \times\left[\mathbf{W}^{1,2} \cap \mathbf{H}_{0}(\operatorname{div} ; \Omega)\right]$ be the solution of

$$
\begin{aligned}
\left(d_{t} \mathbf{u}^{n}, \boldsymbol{\xi}\right)+\frac{1}{\operatorname{Re}}\left(\nabla \mathbf{u}^{n}, \nabla \boldsymbol{\xi}\right)+\left(\left(\mathbf{u}^{n-1} \cdot \nabla\right) \mathbf{u}^{n}, \boldsymbol{\xi}\right) & \\
+S\left(\mathbf{b}^{n} \times \operatorname{curl} \mathbf{b}^{n}, \boldsymbol{\xi}\right)+\left(\nabla p^{n}, \boldsymbol{\xi}\right) & =\left(\mathbf{g}^{n}, \boldsymbol{\xi}\right) \quad \forall \boldsymbol{\xi} \in \mathbf{W}_{0}^{1,2}(\Omega), \\
\left(d_{t} \mathbf{b}^{n}, \boldsymbol{\psi}\right)+\frac{1}{\operatorname{Re}_{m}}\left(\operatorname{curl} \mathbf{b}^{n}, \operatorname{curl} \psi\right)+\gamma\left(\operatorname{div} \mathbf{b}^{n}, \operatorname{div} \boldsymbol{\psi}\right) & \\
-\left(\mathbf{u}^{n} \times \mathbf{b}^{n}, \operatorname{curl} \boldsymbol{\psi}\right) & =0 \quad \forall \boldsymbol{\psi} \in \mathbf{H}_{0}(\operatorname{div} ; \Omega) \cap \mathbf{W}^{1,2}(\Omega) .
\end{aligned}
$$

Similar to studies for Scheme A, iterates $\left\{\left(\mathbf{u}_{A}^{n}, p_{A}^{n}, \mathbf{b}_{A}^{n}\right)\right\}_{n=1}^{N}$ exist, and verify the discrete energy estimate from Lemma 3.1. A lengthy, but elementary analysis controls implicit time discretization effects $\left(\mathbf{e}_{\varphi}^{n}:=\varphi\left(t_{n}, \cdot\right)-\varphi_{A}^{n}\right)$,

$$
\frac{1}{2} \max _{1 \leq n \leq N}\left[\left\|\mathbf{e}_{\mathbf{u}}^{n}\right\|_{\mathbf{L}^{2}}^{2}+\left\|\mathbf{e}_{\mathbf{b}}^{n}\right\|_{\mathbf{L}^{2}}^{2}\right]+\frac{k^{2}}{2} \sum_{n=1}^{N}\left[\left\|d_{t} \mathbf{e}_{\mathbf{u}}^{n}\right\|_{\mathbf{L}^{2}}^{2}+\left\|d_{t} \mathbf{e}_{\mathbf{b}}^{n}\right\|_{\mathbf{L}^{2}}^{2}\right]+k \sum_{n=1}^{N}\left[\left\|\nabla \mathbf{e}_{\mathbf{u}}^{n}\right\|_{\mathbf{L}^{2}}^{2}+\left\|\nabla \mathbf{e}_{\mathbf{b}}^{n}\right\|_{\mathbf{L}^{2}}^{2}\right] \leq C k^{2},
$$

where $C>0$ depends on (4.5), and results from Taylor's expansion, regularity properties (4.5) of the strong solution of (1.1)-(1.5), cancellation of coupling terms in (4.9) and (4.10) if correspondingly stated for $\mathbf{e}_{\mathbf{u}}$ resp. $\mathbf{e}_{\mathbf{u}}$ instead of $\mathbf{u}$ resp. $\mathbf{b}$, as well as discrete Gronwall's inequality. The second sum results from the dissipative character of the implicit Euler scheme, and implies the following bound for the first two terms

$$
\left\|d_{t} \mathbf{u}_{A}^{n}\right\|_{\mathbf{L}^{2}}+\left\|d_{t} \mathbf{b}_{A}^{n}\right\|_{\mathbf{L}^{2}}+\left\|\mathbf{u}_{A}^{n}\right\|_{\mathbf{W}^{2,2}}+\left\|p_{A}^{n}\right\|_{W^{1,2} \cap L_{0}^{2}}+\left\|\mathbf{b}_{A}^{n}\right\|_{\mathbf{W}^{2,2}} \leq C \quad(1 \leq n \leq N) .
$$

The remaining estimates follow from (2.3) and (2.4), where we use the following bounds,

$$
\begin{aligned}
\left\|\left(\mathbf{u}_{A}^{n-1} \cdot \nabla\right) \mathbf{u}_{A}^{n}\right\|_{\mathbf{L}^{2}} & \leq\left[\left\|\mathbf{e}_{\mathbf{u}}^{n-1}\right\|_{\mathbf{L}^{6}}+\left\|\mathbf{u}\left(t_{n-1}, \cdot\right)\right\|_{\mathbf{L}^{6}}\right]\left\|\nabla \mathbf{u}_{A}^{n}\right\|_{\mathbf{L}^{3}} \\
& \leq C(\sqrt{k}+1)\left\|\nabla \mathbf{u}_{A}^{n}\right\|_{\mathbf{L}^{2}}^{1 / 2}\left\|\mathbf{u}_{A}^{n}\right\|_{\mathbf{W}^{2,2}}^{1 / 2} \\
\left\|\mathbf{b}_{A}^{n} \times \operatorname{curl} \mathbf{b}_{A}^{n}\right\|_{\mathbf{L}^{2}} & \leq\left(\left\|\mathbf{e}_{\mathbf{u}}^{n}\right\|_{\mathbf{L}^{6}}+\left\|\mathbf{u}\left(t_{n}, \cdot\right)\right\|_{\mathbf{L}^{6}}\right)\left\|\operatorname{curl} \mathbf{b}_{A}^{n}\right\|_{\mathbf{L}^{3}} \\
& \leq C(\sqrt{k}+1)\left\|\mathbf{b}_{A}^{n}\right\|_{\mathbf{W}^{1,2}}^{1 / 2}\left\|\mathbf{b}_{A}^{n}\right\|_{\mathbf{W}^{2,2}}^{1 / 2} \\
\left\|\operatorname{curl}\left(\mathbf{u}_{A}^{n} \times \mathbf{b}_{A}^{n}\right)\right\|_{\mathbf{L}^{2}} & \leq\left\|\mathbf{u}_{A}^{n}\right\|_{\mathbf{W}^{1,3}}\left(\left\|\mathbf{e}_{\mathbf{b}}^{n}\right\|_{\mathbf{L}^{6}}+\left\|\mathbf{b}\left(t_{n}, \cdot\right)\right\|_{\mathbf{L}^{6}}\right)+\left\|\mathbf{b}_{A}^{n}\right\|_{\mathbf{W}^{1,3}}\left(\left\|\mathbf{e}_{\mathbf{u}}^{n}\right\|_{\mathbf{L}^{6}}+\left\|\mathbf{u}\left(t_{n}, \cdot\right)\right\|_{\mathbf{L}^{6}}\right) \\
& \leq C(\sqrt{k}+1)\left[\left\|\mathbf{u}_{A}^{n}\right\|_{\mathbf{W}^{1,2}}^{1 / 2}\left\|\mathbf{u}_{A}^{n}\right\|_{\mathbf{W}^{2,2}}^{1 / 2}+\left\|\mathbf{b}_{A}^{n}\right\|_{\mathbf{W}^{1,2}}^{1 / 2}\left\|\mathbf{b}_{A}^{n}\right\|_{\mathbf{W}^{2,2}}^{1 / 2}\right]
\end{aligned}
$$

In order to show $k \sum_{1 \leq n \leq N}\left[\left\|d_{t} \mathbf{u}^{n}\right\|_{\mathbf{W}^{1,2}}^{2}+\left\|d_{t} \mathbf{b}^{n}\right\|_{\mathbf{W}^{1,2}}^{2}\right] \leq C$, we use again the error estimates above, for example

$$
k \sum_{n=1}^{N}\left\|\nabla d_{t} \mathbf{u}_{A}^{n}\right\|_{\mathbf{L}^{2}}^{2} \leq k \sum_{n=1}^{N}\left[\left\|\nabla d_{t} \mathbf{e}_{\mathbf{u}}^{n}\right\|_{\mathbf{L}^{2}}^{2}+\left\|\nabla d_{t} \mathbf{u}\left(t_{n}, \cdot\right)\right\|_{\mathbf{L}^{2}}^{2}\right] \leq \frac{3}{k} \sum_{n=1}^{N}\left\|\nabla \mathbf{e}_{\mathbf{u}}^{n}\right\|_{\mathbf{L}^{2}}^{2}+C \leq 2 C .
$$

Properties for pressure iterates given in (P1), (P2) use standard stability properties of the div-operator. Hence properties (P1) and (P2) hold for (4.9)-(4.10).

Step 2: Decoupling of the implicit discretization (4.9)-(4.10). We change the coupling term in (4.9) to $S\left(\mathbf{b}^{n-1} \times\right.$ curl $\left.\mathbf{b}^{n}, \boldsymbol{\xi}\right)$, and in (4.10) to $\left(\mathbf{u}^{n-1} \times \mathbf{b}^{n-1}, \operatorname{curl} \boldsymbol{\psi}\right)$; solutions of the corresponding scheme are referred to as $\left\{\left(\mathbf{u}_{B}^{n}, p_{B}^{n}, \mathbf{b}_{B}^{n}\right)\right\} \in \mathbf{J} \times W^{1,2}(\Omega) \times\left[\mathbf{W}^{1,2}(\Omega) \cap \mathbf{H}_{0}(\operatorname{div} ; \Omega)\right]$.

We may proceed similar to the previous step to validate properties (P1) and (P2) for $\left\{\left(\mathbf{u}_{B}^{n}, p_{B}^{n}, \mathbf{b}_{B}^{n}\right)\right\}_{n=1}^{N}$ : in order to verify the discrete energy inequality (3.17), we use integration by parts in 
the third line, and Sobolev inequalities in the fourth line of (3.16) to obtain

$$
\begin{aligned}
&\left(\mathbf{B}^{n-1}\right.\left.\times \operatorname{curl} \mathbf{B}^{n-1}, \mathbf{U}^{n}\right)-\left(\mathbf{U}^{n-1} \times \mathbf{B}^{n-1}, \operatorname{curl} \mathbf{B}^{n}\right) \geq-C k\left[\left\|\mathbf{B}^{n-1}\right\|_{\mathbf{W}^{2,2}}\left\|\operatorname{curl} \mathbf{B}^{n-1}\right\|_{\mathbf{L}^{2}}\left\|d_{t} \mathbf{U}^{n}\right\|_{\mathbf{L}^{2}}\right. \\
&\left.+\left(\left\|\mathbf{U}^{n-1}\right\|_{\mathbf{W}^{1,2}}\left\|\mathbf{B}^{n-1}\right\|_{\mathbf{W}^{2,2}}+\left\|\mathbf{B}^{n-1}\right\|_{\mathbf{W}^{1,2}}\left\|\mathbf{U}^{n-1}\right\|_{\mathbf{W}^{2,2}}\right)\left\|d_{t} \mathbf{B}^{n}\right\|_{\mathbf{L}^{2}}\right] \\
& \geq-\frac{k}{8}\left[\left\|d_{t} \mathbf{U}^{n}\right\|_{\mathbf{L}^{2}}^{2}+\frac{1}{\operatorname{Re}_{m}}\left\|d_{t} \mathbf{B}^{n}\right\|_{\mathbf{L}^{2}}^{2}\right]+C k\left[\left\|\mathbf{U}^{n-1}\right\|_{\mathbf{W}^{2,2}}^{2}+\left\|\mathbf{B}^{n-1}\right\|_{\mathbf{W}^{2,2}}^{2}\right] \\
& \quad \times\left[\left\|\mathbf{B}^{n-1}\right\|_{\mathbf{W}^{1,2}}^{2}+\left\|\mathbf{u}^{n-1}\right\|_{\mathbf{W}^{1,2}}^{2}\right]
\end{aligned}
$$

Now, for every $1 \leq n \leq N$ the error estimate (4.11) carries over to $\left(\mathbf{u}_{B}^{n}, p_{B}^{n}, \mathbf{b}_{B}^{n}\right)$, as well as its consequences (4.12), and an inductive argument then settles (3.17) for them. The remaining properties of (P1) and (P2) now easily follow again.

Step 3: Chorin's projection method. For every $1 \leq n \leq N$, let $\left(\mathbf{u}_{C}^{n}, p_{C}^{n}, \mathbf{b}_{C}^{n}\right) \in \mathbf{W}_{0}^{1,2}(\Omega) \times\left[W^{1,2}(\Omega) \cap L_{0}^{2}(\Omega)\right] \times$ $\left[\mathbf{W}^{1,2}(\Omega) \cap \mathbf{H}_{0}(\operatorname{div} ; \Omega)\right]$ be the solution of

$$
\begin{array}{r}
\left(d_{t} \mathbf{u}_{C}^{n}, \boldsymbol{\xi}\right)+\frac{1}{\operatorname{Re}}\left(\nabla \mathbf{u}_{C}^{n}, \nabla \boldsymbol{\xi}\right)+\left(\left(\mathbf{u}_{C}^{n-1} \cdot \nabla\right) \mathbf{u}_{C}^{n}, \boldsymbol{\xi}\right)+\frac{1}{2}\left(\left(\operatorname{div} \mathbf{u}_{C}^{n-1}\right) \mathbf{u}_{C}^{n}, \boldsymbol{\xi}\right) \\
+S\left(\mathbf{b}_{B}^{n-1} \times \operatorname{curl}_{B}^{n-1}, \boldsymbol{\xi}\right)+\left(\nabla p_{C}^{n-1}, \boldsymbol{\xi}\right)=\left(\mathbf{g}^{n}, \boldsymbol{\xi}\right) \quad \forall \boldsymbol{\xi} \in \mathbf{W}_{0}^{1,2}(\Omega) \\
\left(\operatorname{div} \mathbf{u}_{C}^{n}, \chi\right)+k\left(\nabla p_{C}^{n}, \nabla \chi\right)=0 \quad \forall \chi \in W^{1,2}(\Omega) \cap L_{0}^{2}(\Omega) \\
\left(d_{t} \mathbf{b}_{C}^{n}, \boldsymbol{\psi}\right)+\frac{1}{\operatorname{Re}_{m}}\left(\operatorname{curl} \mathbf{b}_{C}^{n}, \operatorname{curl} \boldsymbol{\psi}\right)+\gamma\left(\operatorname{div} \mathbf{b}_{C}^{n}, \operatorname{div} \boldsymbol{\psi}\right) \\
-\left(\mathbf{u}_{C}^{n-1} \times \mathbf{b}_{C}^{n-1}, \operatorname{curl} \boldsymbol{\psi}\right)=0 \quad \forall \boldsymbol{\psi} \in \mathbf{H}_{0}(\operatorname{div} ; \Omega) \cap \mathbf{W}^{1,2}(\Omega)
\end{array}
$$

We have $\mathbf{b}_{B}^{n-1} \times \operatorname{curl}_{B}^{n-1} \in \mathbf{W}^{1,2}(\Omega)$ for every $1 \leq n \leq N$; by Lemma 2.4 , and using $\mathbf{e}_{\varphi}^{n}:=\varphi_{B}^{n}-\varphi_{C}^{n}$, we conclude that

$$
\frac{1}{2} \max _{1 \leq n \leq N}\left\|\mathbf{e}_{\mathbf{u}}^{n}\right\|_{\mathbf{L}^{2}}^{2}+\frac{k^{2}}{2} \sum_{n=1}^{N}\left[\left\|d_{t} \mathbf{e}_{\mathbf{u}}^{n}\right\|_{\mathbf{L}^{2}}^{2}+2\left\|\nabla e_{p}^{n}\right\|_{\mathbf{L}^{2}}^{2}\right]+k \sum_{n=1}^{N}\left\|\nabla \mathbf{e}_{\mathbf{u}}^{n}\right\|_{\mathbf{L}^{2}}^{2} \leq C k^{2} .
$$

Therefore, $\left\|p^{n}\right\|_{W^{1,2} \cap L_{0}^{2}} \leq C$ for all $1 \leq n \leq N$, and because of (4.15), we so we have similar to (4.12),

$$
\left\|d_{t} \mathbf{u}_{C}^{n}\right\|_{\mathbf{L}^{2}}+\left\|p_{C}^{n}\right\|_{W^{1,2} \cap L_{0}^{2}}+\left\|\mathbf{u}_{C}^{n}\right\|_{\mathbf{W}^{2,2}} \leq C \quad(1 \leq n \leq N)
$$

where the latter result now follows from (4.14), and regularity theory for elliptic problems.

In order to verify results which correspond to those in (4.17), (4.18) for $\left\{\mathbf{b}_{C}^{n}\right\}_{n} \subset\left[\mathbf{W}^{1,2}(\Omega) \cap \mathbf{H}_{0}(\operatorname{div} ; \Omega)\right]$, we use (4.16) to control $\left\{\mathbf{e}_{\mathbf{b}}^{n}\right\}_{n} \subset\left[\mathbf{W}^{1,2}(\Omega) \cap \mathbf{H}_{0}(\operatorname{div} ; \Omega)\right]$ : by elementary calculations, on using

$$
\begin{aligned}
\left(\mathbf{u}_{B}^{n-1} \times \mathbf{b}_{B}^{n-1}-\mathbf{u}_{C}^{n-1} \times \mathbf{b}_{C}^{n-1}, \operatorname{curl} \mathbf{e}_{\mathbf{b}}^{n}\right) & =\left(\mathbf{u}_{C}^{n-1} \times \mathbf{e}_{\mathbf{b}}^{n-1}, \operatorname{curl} \mathbf{e}_{\mathbf{b}}^{n}\right)+\left(\mathbf{e}_{\mathbf{u}}^{n-1} \times \mathbf{b}_{B}^{n-1}, \operatorname{curl}, \mathbf{e}_{\mathbf{b}}^{n}\right) \\
& \leq\left(\left\|\mathbf{u}_{C}^{n-1}\right\|_{\mathbf{L}^{\infty}}+\left\|\mathbf{b}_{B}^{n-1}\right\|_{\mathbf{L}^{\infty}}\right)\left\|\mathbf{e}_{\mathbf{b}}^{n-1}\right\|_{\mathbf{L}^{2}}\left\|\operatorname{curl} \mathbf{e}_{\mathbf{b}}^{n}\right\|_{\mathbf{L}^{2}}
\end{aligned}
$$

Sobolev's and Young's inequality, as well as a priori bounds from the previous step, this implies properties (P1) and (P2) for $\left\{\left(\mathbf{u}_{C}^{n}, p_{C}^{n}, \mathbf{b}_{C}^{n}\right)\right\}_{1 \leq n \leq N}$.

Step 4: Recoupling effects caused by Chorin's projection method. For every $1 \leq n \leq N$, let $\left(\mathbf{u}_{D}^{n}, p_{D}^{n}, \mathbf{b}_{D}^{n}\right) \in \mathbf{W}_{0}^{1,2}(\Omega) \times\left[W^{1,2}(\Omega) \cap L_{0}^{2}(\Omega)\right] \times\left[\mathbf{W}^{1,2}(\Omega) \cap \mathbf{H}_{0}(\operatorname{div} ; \Omega)\right]$ be the solution of a slight modification of (4.14)-(4.16), where the coupling term in (4.14) replaced by $S\left(\mathbf{b}_{D}^{n-1} \times \operatorname{curl} \mathbf{b}_{B}^{n-1}, \boldsymbol{\xi}\right)$. Let $\mathbf{e}_{\varphi}^{n}:=\varphi_{C}^{n}-\varphi_{D}^{n}$, 
then the equations for $\left(\mathbf{e}_{\mathbf{u}}^{n}, e_{p}^{n}, \mathbf{e}_{\mathbf{b}}^{n}\right) \in \mathbf{W}_{0}^{1,2}(\Omega) \times\left[W^{1,2}(\Omega) \cap L_{0}^{2}(\Omega)\right] \times\left[\mathbf{W}^{1,2}(\Omega) \cap \mathbf{H}_{0}(\operatorname{div} ; \Omega)\right] \mathrm{read}$

$$
\begin{aligned}
&\left(d_{t} \mathbf{e}_{\mathbf{u}}^{n}, \boldsymbol{\xi}\right)+\frac{1}{\operatorname{Re}}\left(\nabla \mathbf{e}_{\mathbf{u}}^{n}, \nabla \boldsymbol{\xi}\right)+\left(\left[\mathbf{u}_{C}^{n-1} \cdot \nabla\right] \mathbf{e}_{\mathbf{u}}^{n}, \boldsymbol{\xi}\right)-\left(\left[\mathbf{e}_{\mathbf{u}}^{n-1} \cdot \nabla\right] \mathbf{e}_{\mathbf{u}}^{n}, \boldsymbol{\xi}\right)-\left(\left[\mathbf{e}_{\mathbf{u}}^{n-1} \cdot \nabla\right] \mathbf{u}_{C}^{n}, \boldsymbol{\xi}\right) \\
&+ \frac{1}{2}\left[\left(\left(\operatorname{div} \mathbf{u}_{C}^{n-1}\right) \mathbf{e}_{\mathbf{u}}^{n}, \boldsymbol{\xi}\right)+\left(\left(\operatorname{div} \mathbf{e}_{\mathbf{u}}^{n-1}\right) \mathbf{e}_{\mathbf{u}}^{n}, \boldsymbol{\xi}\right)+\left(\left(\operatorname{div} \mathbf{e}_{\mathbf{u}}^{n-1}\right) \mathbf{u}_{C}^{n}, \boldsymbol{\xi}\right)\right] \\
&+S\left[\left(\mathbf{b}_{C}^{n-1} \times \operatorname{curl} \mathbf{e}_{\mathbf{b}}^{n-1}, \boldsymbol{\xi}\right)-\left(\mathbf{e}_{\mathbf{b}}^{n-1} \times \operatorname{curl} \mathbf{e}_{\mathbf{b}}^{n-1}, \boldsymbol{\xi}\right)-\left(\mathbf{e}_{\mathbf{b}}^{n-1} \times \operatorname{curl} \mathbf{u}_{C}^{n-1}, \boldsymbol{\xi}\right)\right]+\left(\nabla e_{p}^{n-1}, \boldsymbol{\xi}\right)= \\
&-S\left[\left(\mathbf{b}_{B}^{n-1} \times \operatorname{curl}\left[\mathbf{b}_{B}^{n-1}-\mathbf{b}_{C}^{n-1}\right], \boldsymbol{\xi}\right)-\left(\left[\mathbf{b}_{B}^{n-1}-\mathbf{b}_{C}^{n-1}\right] \times \operatorname{curl}\left[\mathbf{b}_{B}^{n-1}-\mathbf{b}_{C}^{n-1}\right], \boldsymbol{\xi}\right)\right. \\
&\left.-\left(\left[\mathbf{b}_{B}^{n-1}-\mathbf{b}_{C}^{n-1}\right] \times \operatorname{curl} \mathbf{u}_{B}^{n-1}, \boldsymbol{\xi}\right)\right] \quad \forall \boldsymbol{\xi} \in \mathbf{W}_{0}^{1,2}(\Omega), \\
&\left(\operatorname{div} \mathbf{e}_{\mathbf{u}}^{n}, \chi\right)+k\left(\nabla e_{p}^{n}, \nabla \chi\right)=0 \quad \forall \chi \in W^{1,2}(\Omega) \cap L_{0}^{2}(\Omega), \\
&\left(d_{t} \mathbf{e}_{\mathbf{b}}^{n}, \boldsymbol{\psi}\right)+\frac{1}{\operatorname{Re}_{m}}\left(\operatorname{curl} \mathbf{e}_{\mathbf{b}}^{n}, \operatorname{curl} \boldsymbol{\psi}\right)+\gamma\left(\operatorname{div} \mathbf{e}_{\mathbf{b}}^{n-1}, \operatorname{div} \boldsymbol{\psi}\right)-\left(\mathbf{u}_{C}^{n-1} \times \mathbf{e}_{\mathbf{b}}^{n-1}, \operatorname{curl} \boldsymbol{\psi}\right) \\
&+\left(\mathbf{e}_{\mathbf{u}}^{n-1} \times \mathbf{e}_{\mathbf{b}}^{n-1}, \operatorname{curl} \boldsymbol{\psi}\right)-\left(\mathbf{e}_{\mathbf{u}}^{n-1} \times \mathbf{b}_{C}^{n-1}, \operatorname{curl} \boldsymbol{\psi}\right)=0 \quad \forall \boldsymbol{\psi} \in \mathbf{H}_{0}(\operatorname{div} ; \Omega) \cap \mathbf{W}^{1,2}(\Omega) .
\end{aligned}
$$

We choose $(\boldsymbol{\xi}, \chi, \boldsymbol{\psi})=\left(\mathbf{e}_{\mathbf{u}}^{n}, e_{p}^{n}, \mathbf{e}_{\mathbf{b}}^{n}\right)$, add the three equations, and sum over all time steps $1 \leq n \leq N$. Because of properties (P1) and (P2) for $\left\{\left(\mathbf{u}_{C}^{n}, p_{C}^{n}, \mathbf{b}_{C}^{n}\right)\right\}_{n=1}^{N}$, most of the terms are simple to control, and we focus here on only two which cause some difficulties. We start with

$$
\left(\mathbf{e}_{\mathbf{b}}^{n-1} \times \operatorname{curl} \mathbf{e}_{\mathbf{b}}^{n-1}, \mathbf{e}_{\mathbf{u}}^{n}\right)-\left(\mathbf{e}_{\mathbf{b}}^{n-1} \times \mathbf{e}_{\mathbf{u}}^{n-1}, \operatorname{curl} \mathbf{e}_{\mathbf{b}}^{n}\right)
$$

Similar to (4.13), we may employ cancellation of the terms for equal indices, and a priori estimates (P2) for $\left\{\left(\mathbf{u}_{D}^{n}, p_{D}^{n}, \mathbf{b}_{D}^{n}\right)\right\}_{n=1}^{N}$ which are available if the above error analysis is embedded into an inductive argument.

The second problematic term is $\left(\nabla e_{p}^{n-1}, \mathbf{e}_{\mathbf{u}}^{n}\right)=k\left(\nabla e_{p}^{n}, \nabla e_{p}^{n-1}\right)$, which can be restated as follows,

$$
\begin{aligned}
k\left(\nabla e_{p}^{n}, \nabla e_{p}^{n-1}\right) & =k\left\|\nabla e_{p}^{n}\right\|_{\mathbf{L}^{2}}^{2}-k^{2}\left(\nabla e_{p}^{n}, \nabla d_{t} e_{p}^{n}\right) \\
& =k\left\|\nabla e_{p}^{n}\right\|_{\mathbf{L}^{2}}^{2}-k\left(d_{t} \mathbf{e}_{\mathbf{u}}^{n}, \nabla e_{p}^{n}\right) \geq k\left[\frac{1}{8}\left\|\nabla e_{p}^{n}\right\|_{\mathbf{L}^{2}}^{2}-\frac{2}{7}\left\|d_{t} \mathbf{e}_{\mathbf{u}}^{n}\right\|_{\mathbf{L}^{2}}^{2}\right],
\end{aligned}
$$

where we employ again the quasi-compressibility constraint. Thanks to the dissipative character of the implicit Euler method, the last term may again be control by $\left(d_{t} \mathbf{e}_{\mathbf{u}}^{n}, \mathbf{e}_{\mathbf{u}}^{n}\right)=\frac{1}{2} d_{t}\left\|\mathbf{e}_{\mathbf{u}}^{n}\right\|_{\mathbf{L}^{2}}^{2}+\frac{k}{2}\left\|d_{t} \mathbf{e}_{\mathbf{u}}^{n}\right\|_{\mathbf{L}^{2}}^{2}$. As a consequence, we may conclude (P1), and (P2) then easily follows from the previous steps.

The proof of Theorem 4.1 is an immediate consequence of Lemma 4.1.

Proof of Theorem 4.1. Lemma 4.1 asserts the approximation property (P2) for solutions $\left\{\left(\mathbf{u}^{n}, p^{n}, \mathbf{b}^{n}\right)\right\}_{n=1}^{N}$ of (4.6)-(4.8). By the method of proof of Lemma 4.1, we may use it to validate property (P2) for solutions of (4.6)-(4.8) as well. 
In order to verify Theorem 4.1, it remains to control spatial discretization effects; for this purpose we subtract equations (4.1)-(4.3) from (4.6)-(4.8). By conformity of the finite element discretization, we obtain $\left(\left(\mathbf{E}_{\mathbf{u}}^{n}, E_{p}^{n}, \mathbf{E}_{\mathbf{u}}^{n}\right):=\left(\mathbf{u}^{n}-\tilde{\mathbf{U}}^{n}, p^{n}-P^{n}, \mathbf{b}^{n}-\mathbf{B}^{n}\right)\right)$

$$
\begin{aligned}
& \left(d_{t} \mathbf{E}_{\mathbf{u}}^{n}, \boldsymbol{\Xi}\right)+\frac{1}{\operatorname{Re}}\left(\nabla \mathbf{E}_{\mathbf{u}}^{n}, \nabla \boldsymbol{\Xi}\right)+\left(\left[\mathbf{u}^{n-1} \cdot \nabla\right] \mathbf{E}_{\mathbf{u}}^{n}, \Xi\right)-\left(\left[\mathbf{E}_{\mathbf{u}}^{n-1} \cdot \nabla\right] \mathbf{E}_{\mathbf{u}}^{n}, \Xi\right)-\left(\left[\mathbf{E}_{\mathbf{u}}^{n-1} \cdot \nabla\right] \mathbf{u}^{n}, \Xi\right) \\
& +\frac{1}{2}\left[\left(\left(\operatorname{div} \mathbf{u}^{n-1}\right) \mathbf{E}_{\mathbf{u}}^{n}, \Xi\right)+\left(\left(\operatorname{div} \mathbf{E}_{\mathbf{u}}^{n-1}\right) \mathbf{E}_{\mathbf{u}}^{n}, \Xi\right)+\left(\left(\operatorname{div} \mathbf{E}_{\mathbf{u}}^{n-1}\right) \mathbf{u}^{n}, \Xi\right)\right] \\
& +S\left[\left(\mathbf{b}^{n-1} \times \operatorname{curl} \mathbf{E}_{\mathbf{b}}^{n-1}, \Xi\right)-\left(\mathbf{E}_{\mathbf{b}}^{n-1} \times \operatorname{curl} \mathbf{E}_{\mathbf{b}}^{n-1}, \Xi\right)\right. \\
& \left.-\left(\mathbf{E}_{\mathbf{b}}^{n-1} \times \operatorname{curl} \mathbf{u}^{n-1}, \Xi\right)\right]+\left(\nabla E_{p}^{n-1}, \boldsymbol{\Xi}\right)=0 \quad \forall \boldsymbol{\Xi} \in \mathbf{V}_{h} \\
& \left(\operatorname{div} \mathbf{E}_{\mathbf{u}}^{n}, \Lambda\right)+k\left(\nabla E_{p}^{n}, \nabla \Lambda\right)=0 \quad \forall \Lambda \in \tilde{L}_{h}, \\
& \left(d_{t} \mathbf{E}_{\mathbf{b}}^{n}, \boldsymbol{\Psi}\right)+\frac{1}{\operatorname{Re}_{m}}\left(\operatorname{curl} \mathbf{E}_{\mathbf{b}}^{n}, \operatorname{curl} \Psi\right)+\gamma\left(\operatorname{div} \mathbf{E}_{\mathbf{b}}^{n-1}, \operatorname{div} \boldsymbol{\Psi}\right)-\left(\mathbf{u}^{n-1} \times \mathbf{E}_{\mathbf{b}}^{n-1}, \operatorname{curl} \boldsymbol{\Psi}\right) \\
& +\left(\mathbf{E}_{\mathbf{u}}^{n-1} \times \mathbf{E}_{\mathbf{b}}^{n-1}, \operatorname{curl} \Psi\right)-\left(\mathbf{E}_{\mathbf{u}}^{n-1} \times \mathbf{b}^{n-1}, \operatorname{curl} \Psi\right) \quad \forall \boldsymbol{\psi} \in \tilde{\mathbf{C}}_{h} .
\end{aligned}
$$

Let $(\Xi, \Lambda, \Psi)=\left(\mathbf{P}_{\mathbf{W}^{1,2}} \mathbf{E}_{\mathbf{u}}^{n}, \mathrm{P}_{W^{1,2} \cap L_{0}^{2}} E_{p}^{n}, \mathbf{P}_{\mathbf{W}^{1,2}} E_{\mathbf{u}}^{n}\right) \in \mathbf{V}_{h} \times \tilde{L}_{h} \times \tilde{\mathbf{C}}_{h}$. A standard error analysis now shows assertion Theorem 4.1 .

Remark 4.1. (1) The stabilized finite element method introduced in [11] to solve the stationary incompressible MHD system is related to the Scheme C, in the sense that the (consistent) stabilization procedure in [11] is based on the quasi-compressibility constraint $(\epsilon>0)$

$$
\operatorname{div} \mathbf{u}-\epsilon \Delta=\epsilon \mathbf{h} \quad \text { in } \Omega,
$$

for some $\mathbf{h} \equiv \mathbf{h}(\mathbf{u}, \mathbf{b})$, and $\epsilon=\mathcal{O}\left(h^{2}\right)$. In the context of Scheme $\mathrm{C}$, we have $\mathbf{h} \equiv \mathbf{0}$, and $\epsilon=\mathcal{O}(k)-$ which leads to the mesh-constraint $k \geq C h^{2}$ to apply the results from [19] to circumvent the discrete LBB constraint, and validate well-posedness of the discrete scheme, as well as optimal convergence behavior.

(2) A well-known drawback of projection methods are arising non-physical boundary layers for pressure iterates; we refer to $[22,24]$ where modified methods are introduced and studied, which enforce the incompressibility constraint by projection, and are exempted from this deficiency.

(3) Another statement of (1.1)-(1.3) is the Helmholtz formulation

$$
\begin{aligned}
\mathbf{u}_{t}+(\mathbf{u} \cdot \nabla) \mathbf{u}-\frac{1}{\operatorname{Re}} \Delta \mathbf{u}+\nabla\left(p+\frac{S}{2}|\mathbf{b}|^{2}\right) & =S(\mathbf{b} \cdot \nabla) \mathbf{b}+\mathbf{g} \\
\operatorname{div} \mathbf{u}=0 \quad \operatorname{div} \mathbf{b} & =0 \\
\mathbf{b}_{t}-\frac{1}{\operatorname{Re}_{m}} \Delta \mathbf{b}+(\mathbf{u} \cdot \nabla) \mathbf{b}-(\mathbf{b} \cdot \nabla) \mathbf{u}+\nabla q & =\mathbf{0},
\end{aligned}
$$

where $q \in L_{0}^{2}(\Omega)$ is a Lagrange multiplier to allow for solenoidal fields $\mathbf{b}: \Omega_{T} \rightarrow \mathbb{R}^{3}$. In [23], Sections 6 and 7 , different quasi-compressibility strategies are studied which mimic corresponding projection methods in the context of splitting temporal discretization. However, as is outlined in [12], Section 3.6, the Helmholtz formulation is not well-suited to implement the natural boundary conditions (1.4), which is the reason to better discretize (1.1)-(1.5) directly. 


\section{Conclusions}

It is well-known that global weak solutions for equations (1.1)-(1.5) and $\Omega \subset \mathbb{R}^{3}$ bounded exist for a general context of data, while existence of strong solutions is only locally known, and requires a restricted set of data. In this paper, we propose four different fully discrete schemes which reflect this problematic nature of the problem:

(1) Weak solutions in general polyhedral domains $\Omega \subset \mathbb{R}^{3}$ are approximated by Scheme A, and certain decoupling strategies (Algorithm A and Scheme B), where $\mathbf{H}(\operatorname{curl} ; \Omega)$-conforming edge elements for the magnetic field, and a discrete energy principle are crucial tools to verify convergence of iterates in the sense of existing subsequences which converge to weak solutions of (1.1)-(1.5) for vanishing discretization parameters.

(2) Strong solutions in regular domains $\Omega \subset \mathbb{R}^{3}$ are approximated by iterates from a time-splitting scheme (Scheme C) which uses nodal finite elements, in the sense of strong convergence with optimal rates.

\section{REFERENCES}

[1] C. Amrouche, C. Bernardi, M. Dauge and V. Girault, Vector potentials in three-dimensional nonsmooth domains. Math. Meth. Appl. Sci. 21 (1998) 823-864.

[2] F. Armero and J.C. Simo, Long-time dissipativity of time-stepping algorithms for an abstract evolution equation with applications to the incompressible MHD and Navier-Stokes equations. Comp. Meth. Appl. Mech. Engrg. 131 (1996) 41-90.

[3] L. Banas and A. Prohl, Convergent finite element discretization of the multi-fluid nonstationary incompressible magnetohydrodynamics equations. (In preparation).

[4] D. Boffi, P. Fernandes, L. Gastaldi and I. Perugia, Computational models of electromagnetic resonators: analysis of edge element approximation. SIAM J. Numer. Anal. 36 (1999) 1264-1290.

[5] S.C. Brenner and L.R. Scott, The mathematical theory of finite element methods. Springer (1994).

[6] L. Cattabriga, Su un problema al contorno relativo al sistemo di equazioni di Stokes. Rend. Sem Mat. Univ. Padova 31 (1961) 308-340.

[7] Z. Chen, Q. Du and J. Zou, Finite element methods with matching and nonmatching meshes for Maxwell equations with discontinuous coefficients. SIAM J. Numer. Anal. 37 (2000) 1542-1570.

[8] A.J. Chorin, Numercial solution of the Navier-Stokes equations. Math. Comp. 22 (1968) 745-762.

[9] M. Costabel and M. Dauge, Weighted regularization of Maxwell equations in polyhedral domains. Numer. Math. 93 (2002) $239-277$.

[10] V. Georgescu, Some boundary value problems for differential forms on compact Riemannian manifolds. Ann. Math. Pura Appl. 122 (1979) 159-198.

[11] J.-F. Gerbeau, A stabilized finite element method for the incompressible magnetohydrodynamic equations. Numer. Math. 87 (2000) 83-111.

[12] J.-F. Gerbeau, C. Le Bris and T. Lelievre, Mathematical methods for the magnetohydrodynamics of liquid crystals. Oxford Science Publication (2006).

[13] V. Girault, R.H. Nochetto and R. Scott, Maximum-norm stability of the finite element Stokes projection. J. Math. Pures Appl. 84 (2005) 279-330.

[14] V. Girault and P.-A. Raviart, Finite element methods for Navier-Stokes equations. Springer (1986).

[15] M.D. Gunzburger, A.J. Meir and J.S. Peterson, On the existence and uniqueness and finite element approximation of solutions of the equations of stationary incompressible magnetohydrodynamics. Math. Comp. 56 (1991) 523-563.

[16] U. Hasler, A. Schneebeli and D. Schötzau, Mixed finite element approximation of incompressible MHD problems based on weighted regularization. Appl. Numer. Math. 51 (2004) 19-45.

[17] J.G. Heywood and R. Rannacher, Finite element solution of the nonstationary Navier-Stokes problem, I. Regularity of solutions and second-order error estimates for spatial discretization. SIAM J. Numer. Anal. 19 (1982) 275-311.

[18] R. Hiptmair, Finite elements in computational electromagnetism. Acta Numer. 11 (2002) 237-339.

[19] T.J.R. Hughes, L.P. Franca and M. Balestra, A new finite element formulation for computational fluid mechanics: V. Circumventing the Babuska-Brezzi condition: A stable Petrov-Galerkin formulation of the Stokes problem accommodating equal order interpolation. Comp. Meth. Appl. Mech. Eng. 59 (1986) 85-99.

[20] F. Kikuchi, On a discrete compactness property for the Nédélec finite elements. J. Fac. Sci. Univ. Tokyo Sec. IA 36 (1989) 479-490.

[21] P. Monk, Finite element methods for Maxwell's equations. Oxford University Press, New York (2003).

[22] A. Prohl, Projection and quasi-compressibility methods for solving the incompressible Navier-Stokes equations. Teubner-Verlag, Stuttgart (1997).

[23] A. Prohl, On the pollution effect of quasi-compressibility methods in magneto-hydrodynamics and reactive flows. Math. Meth. Appl. Sci. 22 (1999) 1555-1584. 
[24] A. Prohl, On pressure approximation via projection methods for nonstationary incompressible Navier-Stokes equations. SIAM J. Numer. Anal. (to appear).

[25] D. Schötzau, Mixed finite element methods for stationary incompressible magneto-hydrodynamics. Numer. Math. 96 (2004) $771-800$.

[26] M. Sermange and R. Temam, Some mathematical questions related to the MHD equations. Comm. Pure Appl. Math. 36 (1983) 635-664.

[27] R. Temam, Sur l'approximation de la solutoin des equations de Navier-Stokes par la méthode de pas fractionnaires II. Arch. Rat. Mech. Anal. 33 (1969) 377-385.

[28] J. Zhao, Analysis of finite element approximation for time-dependent Maxwell problems. Math. Comp. 73 (2003) $1089-1105$. 